\title{
Effectiveness of VR Head Mounted Displays in Professional Training: A Systematic Review
}

\author{
Sathiya kumar Renganayagalu ${ }^{1,2}$ D $\cdot$ Steven C. Mallam ${ }^{1} \cdot$ Salman Nazir ${ }^{1}$
}

Accepted: 12 December 2020 / Published online: 1 January 2021

(c) The Author(s) 2021

\begin{abstract}
Over the past decade, virtual reality (VR) has re-emerged as a popular technology trend. This is mainly due to the recent investments from technology companies that are improving VR systems while increasing consumer access and interest. Amongst many applications of $\mathrm{VR}$, one area that is particularly promising is for pedagogy. The immersive, experiential learning offered by VR provides new training and learning opportunities driven by the latest versions of affordable, highly immersive and easy to use head mounted display (HMD) systems. VR has been tested as a tool for training across diverse settings with varying levels of success in the past. However, there is a lack of recent review studies that investigates the effectiveness, advantages, limitations, and feasibility of using VR HMDs in training. This review aims to investigate the extent to which VR applications are useful in training, specifically for professional skill and safety training contexts. In this paper, we present the results from a systematic review of the effectiveness of VR-based simulation training from the past 30 years. As a secondary aim, the methodological trends of application and practical challenges of implementing VR in training curriculum were also assessed. The results suggest that there is generally high acceptance amongst trainees for VR-based training regardless of the technology limitations, usability challenges and cybersickness. There is evidence that VR is useful for training cognitive skills, such as spatial memory, learning and remembering procedures and psychomotor skills. VR is also found to be a good alternative where on the job training is either impossible or unsafe to implement. However, many training effectiveness studies reviewed lack experimental robustness due to limited study participants and questionable assessment methods. These results map out the current known strengths and weaknesses of VR HMDs and provide insight into required future research areas as the new era of VR HMD's evolve.
\end{abstract}

Keywords Virtual reality (VR) · Head mounted display (HMD) - Systematic review · Training methods $\cdot$ Effectiveness $\cdot$ Immersive $\cdot$ Education $\cdot$ Pedagogy

Sathiya kumar Renganayagalu

sr@usn.no

1 Training and Assessment Research Group, Department of Maritime Operations, University of South-Eastern Norway, Borre, Norway

2 Department of Virtual and Augmented Reality, Institute for Energy Technology, Halden, Norway 


\section{Introduction}

In recent years, Virtual Reality (VR) has become more prevalent in the consumer marketplace. With the advancements in computing power, display technologies and 3D gaming, Head Mounted Display (HMD) based VR systems are rapidly growing as a consumer product. VR has long been considered as an effective medium for education and training (Pantelidis 2009; Psotka 1995). However, both cost and technological maturity have been major limiting factors for VR proliferation across society, including its utilization for educational and training applications. The introduction of Oculus Rift in 2011 signalled a renewed interest, investment and development in VR HMDs. Since then VR HMDs have become more affordable, easier to use and offer better user experiences. Consumer-grade VR HMDs are now available for less than 500 USD compared to the first commercial HMD VPL eyephone HRX that cost 49,000 USD (IGI consulting 1992) when launched in 1987.

The concept of immersive VR technologies, as we know it today, began in the 1960s when Morton Heilig first introduced Sensorama simulator in 1962 followed by Ivan Sutherland's "The Ultimate Display" concept (Sutherland 1965). Since then, there have been many technological milestones that have expanded the boundaries of VR for individuals to have increased immersive experiences. Throughout the 1970s and 1980s, NASA was the main early adopter of the technology by using VR HMDs in flight simulations and space operations research. The AMES research center at NASA developed VR HMDs for researching telepresence control and telerobotic control for space station operations (Fisher et al. 1987).

The definition of VR has changed throughout these periods based on the context of use and state of technology of that time. Based on the current state of the art, Virtual Reality $(V R)$ is defined as a computer-generated three-dimensional graphical representation of the real or imaginary environment in which users are immersed through a dedicated headset or an array of display walls. In addition, wearable sensors could be worn to provide more sensory cues to the user (e.g. binaural audio, vests and gloves with haptics). VR allows users to interact with a computer-generated world, where the user's natural sensory perceptions are fully/partially replaced with a digital alternative. VR is further described by the following three characteristics (Logan 1998):

Interactivity: The graphical image responds in real-time to the user's commands.

Immersion: The user is drawn into the simulation by sensorial experience.

Imagination: the user's imagination is free to explore the simulated world to see, touch, move and experience things in new ways from new perspectives.

Through this experience, users can find creative solutions to problems and new ways of seeing and doing things (Logan 1998). Although physical immersion is a major characteristic of a VR system, there are other forms of VR systems that are non-immersive (Robertson et al. 1993). Termed as "Desktop VR" or "Fish-Tank VR", this non-immersive VR systems contain computer screens, 3D graphics and interacted through computer input devices. In this study, however, we only focus on the immersive VR and specifically VR HMD systems (Fig. 1), as they are currently the most popular, state of the art VR technology. An HMD is the main hardware component of the current VR technology. Unlike computer screens in the Desktop VR, HMDs often work as both input and output device. Inputs include head motion tracking sensors, gyroscopes and accelerometers. The outputs are the two graphical displays (one for each eye). In VR HMDs the point-of-view of the 

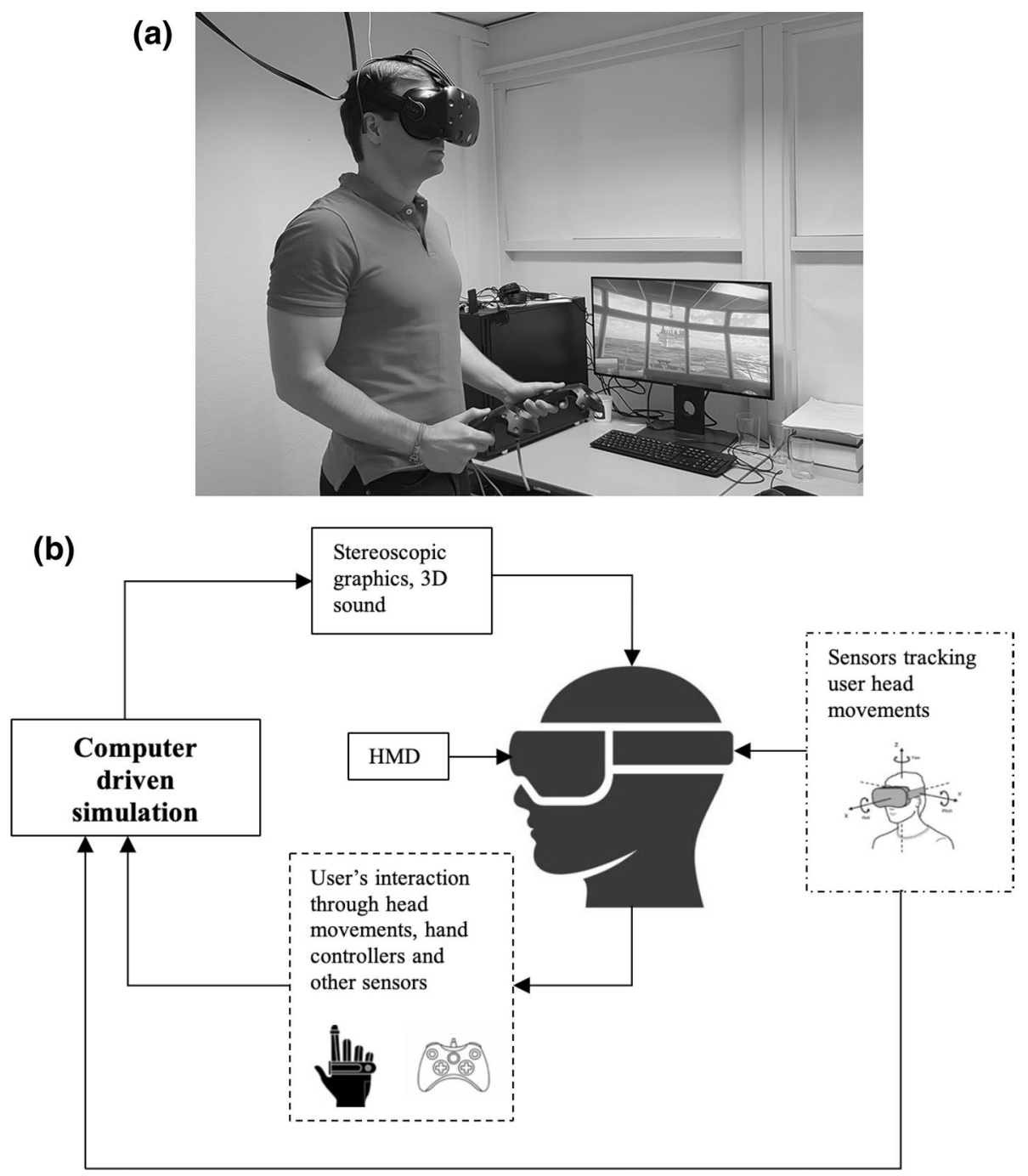

Fig. 1 a Sample VR HMD system, b VR HMD system explained

content in VR is personalized for each user according to their head position. There is also another immersive VR system called Cave Automatic Virtual Environments (CAVE), that consists of a room where all the walls and floor, are projection screens and the system contain motion tracking for tracking user movement. In CAVE systems users wear stereoscopic shutter glasses for the 3D view. Though immersive in nature, these systems are less popular due to the complexity of hardware and the relatively higher costs. 


\section{Background and Related Research}

With the unique characteristics of VR, Dalgarno and Lee (2010) argue that it offers unique affordances for learning, such as representational fidelity, learner interaction and sense of presence (Dalgarno and Lee 2010). There is interest in VR technology for learning because of the assumption that with these affordances of VR, what has been learned in the virtual environment can be transferred to real conditions.

There are many studies and publications that report the benefits, limitations, effects, guidelines and challenges of using VR in education (Farra et al. 2015; Fowler 2015; Pantelidis 2009; Psotka 1995). The key added value of VR lies in the immersion offered by it (Jelfs and Whitelock 2000; Psotka 1995). "Immersion" or "Presence" felt by the users, is the sense of being present in the simulated virtual environment (Witmer and Singer 1998). Stevens and Kincaid (2015) claim that this "sense of being there" enables experiential learning through virtual environments that ultimately leads to positive transfer of knowledge. VR provides a controlled learning environment in which users can navigate, explore, manipulate and inspect the objects and their response in real-time. This explorative learning environment enables users to learn through experimentation. Thus, VR educational applications are best grounded on the constructivist theory of learning (Chen 2010). This constructivist, self-regulated, experiential learning through first-person non-symbolic experiences enabled by VR is the main reason for its value in applying it for education and training (Pantelidis 2009). Reid and Sykes (1999) went further and proposed VR as the ultimate education technology that will change the nature of how students learn. Three-dimensional virtual worlds and their educational uses have long been researched and discussed in the literature (Eschenbrenner et al. 2009; Ludlow 2015). Online virtual platforms, such as 'Second Life' are becoming increasingly prominent and finding interesting applications for learning and education (Minocha and Reeves 2010; Shen and Eder 2009). Considering these potential educational benefits of using VR and the recent advancements in affordable VR technology, the increased interest among educators and researchers to study the technology for educational applications is understandable. There are already a few comprehensive literature reviews that studied the state of the art of VR technology in education (Freina and Ott 2015) and a systematic mapping of educational application of VR HMDs (Jensen and Konradsen 2018). However, these reviews only considered the research that were published after the recent VR boom in 2013 and had a wider and general scope of educational applications of VR. There is currently a gap in the review of literature that focus on professional training applications of VR. Because classroom-based education provides valuable declarative knowledge to the students but practicing complex skills for proficiency is an important part of vocational and professional training. Professional learning settings are inherently different from the classroom based primary education. This type of training is typically delivered through on-the-job experience, simulations (roleplays and computer-based) and practical exercises. Thus, it is important to differentiate it from the rest of education in order to be more specific on the findings.

With VR growing in popularity, there are many efforts from academia and industry to utilize the technology in professional training (Grabowski and Jankowski 2015; Mantovani et al. 2003; Sacks et al. 2013). Hence there is a clear need to analyze how the effectiveness of VR in training has been studied and how results from such studies can inform the future training developments using VR as a training medium. In this review, we are interested to investigate the training applications (refers to the acquisition of skills such as cognitive or psychomotor skills) rather than the educational applications (refers to the acquisition of knowledge or information). 


\subsection{Goals}

The aim of this paper is to make a systematic review of scientific studies on the training effectiveness of VR, to document the available evidence on VR applications and its effects on professional training outcomes. Implementing VR in training still requires development time and resources, so it is not only important to establish the effectiveness of VR in training, but also get inputs for efficient development and use of VR training applications in practice. Another aim of this study is to find if there are any methodological trends in VR application and skills that are more suitable for training using VR. We also aim to document the experimental methods used for testing the training effectiveness of VR that could be referred for input into future studies in this area.

\section{Methods}

This study has been undertaken as a Systematic Literature Review (SLR) based on the guideline from Kitchenham (Kitchenham 2004). A SLR is a method of identifying, evaluating and interpreting available research relevant to a particular research question or topic area (Kitchenham 2004). The main advantage of SLR compared to a normal research review is that it provides a higher degree of confidence about covering the relevant literature, and thus minimizes the subjectivity and bias through reproducible results (Kitchenham et al. 2010). Individual studies that contribute to a systematic review are called primary studies. A systematic review is a form of secondary study.

There are three main reasons for performing a systematic literature review (Kitchenham and Charters 2007).

- To gather and evaluate all existing evidence of a research topic in a rigorous and systematic way

- To identify gaps in current research in order to suggest areas for further improvement

- To summarize and provide background for performing new research activities

The following sub-sections detail the methodology of the SLR process implemented in this study, including the research questions, search strategy, inclusion/exclusion criteria, data extraction and synthesis of results.

\subsection{Research Questions}

The systematic review process consists of 3 stages: (1) Planning the review, (2) Conducting the review and (3) Reporting (Kitchenham 2004). The major differentiating factor of SLR over explorative reviews is the pre-defined protocol and research questions. Defining the scope of the review and answerable questions is an important first step of the SLR process. For defining the scope of the systematic search PICOC framework has been utilized (Booth et al. 2012). Table 1 details the elements defined for this study.

Based on the above-defined scope, the following research questions (RQs) were formulated:

RQ1: What are the benefits, effectiveness and limitations of VR in professional training settings? 
Table 1 PICOC framework

\begin{tabular}{ll}
\hline PICOC Element & Definition \\
\hline Population & Users, trainees in safety-critical areas and professional domains \\
Intervention & Utilizing HMD VR as a training tool or medium \\
Comparison & VR training vs. traditional training or no training \\
Outcomes & Training effectiveness, training transfer and performance indicators \\
Context & Professional training environment with VR HMDs \\
\hline
\end{tabular}

The aim of this question is to identify information and evidence of the training effectiveness of VR-based training. This question answers in what training area and/domains where VR has been applied.

RQ2: How VR is adopted in professional training setups?

The aim of this question is to identify how VR-based training is adopted in different professional training domains. The results obtained will be useful for developing frameworks for future training design using VR.

RQ3: What methodologies are used for assessing the training outcome/effectiveness for VR-based training?

This question aims to map out the methods for measuring training effectiveness in VR based training. The focus is on identifying methodologies and tools for measuring training effectiveness for future studies.

\subsection{Search Process}

Five interdisciplinary research databases were selected for the search (IEEE Xplore, Scopus (ScienceDirect is used for searching full texts), Web of Science, ACM digital library and ERIC). These databases were identified as relevant for education and training, technology applications in learning, psychology and social science. The literature search process was carried out in the month of June 2019.

We performed the search in the above databases using the following search string. The search term for this review combine the terms for virtual reality and training in conjunction with terms for possible outcomes, effectiveness and impacts of such training.

(“Virtual Reality” OR “Immersive VR” OR "Virtual Environment” OR VR)

AND

(Training OR Instruction OR “Training transfer" OR "Skill Acquisition”)

AND

(Impact* OR outcome* OR "skill development” OR affect OR effect*) 


\subsection{Inclusion and Exclusion Criteria}

The inclusion and exclusion criteria for selecting the primary studies were specified according to the SLR methodology. The primary criteria for inclusion were that the studies used immersive and interactive VR environments in three-dimensional (3D) graphics presented with a head-mounted display (HMD) for professional skills and safety training. Considering the research questions, in the general criteria, the time frame for study and relevant type of study were defined.

General Criteria:

- Peer-reviewed studies published between January 1st, 1988 and December 31st, 2018

- Studies that describe the applications and effectiveness of VR in professional training

Specific Criteria:

- Primary studies that compare VR-based training against traditional/typical methods of delivering training

- Primary studies that represent VR as state-of-the-art medium for training

- Studies that use VR HMDs for training professionals

- Secondary studies that quantify VR research in training effectiveness

The following exclusion criteria were defined for this review and studies meeting these criteria are excluded:

- Studies that are not published in English

- Studies that were published before 1988

- Grey literature, ex. white papers, project reports, technical reports

- Books, tutorials and poster publications

- Studies that not include VR HMDs in their testing

- Studies that are not related to/applied for professional training, safety training

\subsection{Data Collection and Extraction}

In the data collection and extraction phase of the review, the documents found in the searching phase were reduced to a final number of documents which were relevant for answering the three research questions. Inclusion and Exclusion criteria were utilized to screen the documents further.

The data extracted from each article are:

- Bibliographic information of the publication

- The application domain of the study

- The population of the study

- Type of training/intervention in the study

- The main research questions of the study and outcomes

- The method used for measuring the outcome

- The metrics used for measuring the outcome

- What are all the objectives/challenges addressed in the study 


\section{Results}

This section presents the results of the review, answering the three research questions based on the extracted data from the 60 studies over a publication period of 30 years. Figure 2 presents the selection criteria processes based on the pre-established framework.

The initial high number of hits for the search string was due to the fact that the term Virtual Reality has been widely used for different applications and contexts. The search was further reduced to the application of HMD VR technology and in training contexts. Documents that describe just the concept or technology application without experiments, surgical simulators that use the term VR but highly purpose-built with surgical tools and other non-immersive VR applications were removed. After the application of inclusion, exclusion and quality criteria 60 studies were selected for the final data analysis.

In this study it was not possible to conduct an accurate meta-analysis as the training application domains, training methodology as well as the study design for assessing the effectiveness of VR with the data collection in those studies differed significantly. Overall, the results were synthesised by extracting the main themes under which the findings of this

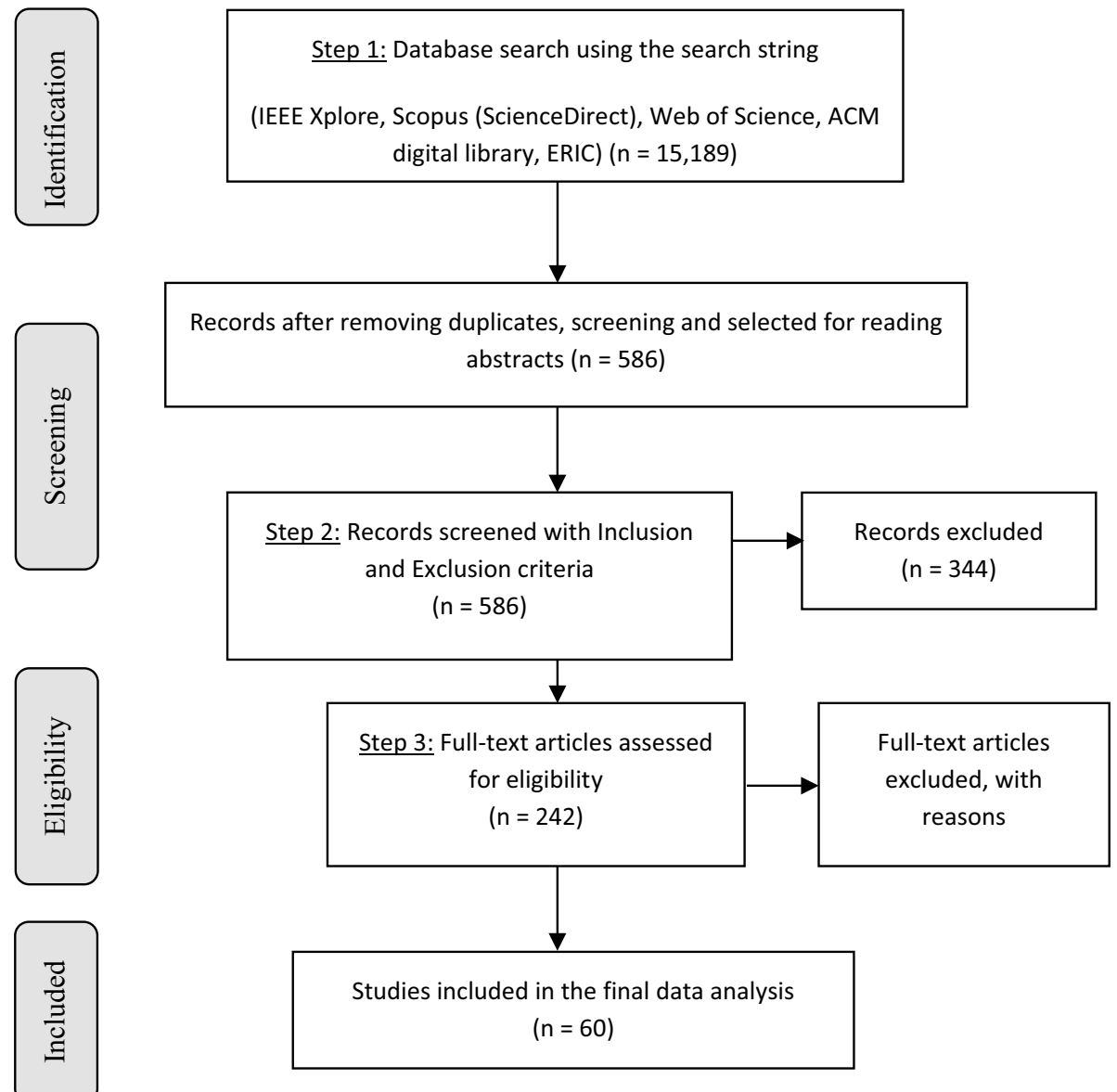

Fig. 2 Results from the search and selection process (PRISMA flow diagram) 
review are identified and presented. The 60 studies included in the final data analysis were categorized and analyzed in the following sub-sections (see Table 2) based on the application domains and types of skills trained.

Although many of the skills trained overlaps between domains and many domains are interrelated (ex: firefighting, safety and emergency preparedness, etc.), these sub-categories help to organize the results.

\subsection{Industrial Training}

Industrial training is one of the major application domains for VR, as it allows to learn and perform simulated hands-on activities in a controlled, safe environment. Out of the 60 reviewed studies, 17 studies $(28.3 \%)$ were on the industrial training applications (see Table 3). The review observed that industrial training applications for VR are mainly focused on procedural skills training for assembly (Boud et al. 1999; Carlson et al. 2015) or maintenance tasks (Bowling et al. 2008; Schroeder et al. 2017). Training has focused on the cognitive aspects of the trainees to recognize and remember parts assembly procedures (Carlson et al. 2015), the sequence of actions (Dwivedi et al. 2018) and orientation of components while carrying out the assembly and maintenance operations. Figure 3 shows the types of training areas for VR applications within the industrial domain. $12 \%$ of the reviewed studies in the industrial domain focused on visual inspection training. (Ragan et al. 2015) claimed field of view (FOV) and visual complexity significantly affected target detection during visual inspection training and found higher FOV led to better performance while higher visual complexity worsened performance. For procedure memorization tasks, enhanced spacial cues such as higher FOV and field of regard in VR HMDs significantly improved training performance (Ragan et al. 2010). In these studies, there is clear evidence that VR affordances such as high immersion benefits in training industrial tasks related to spatial memory skills. When it comes to training effectiveness and user acceptance of the training methodology, almost all reviewed studies reported positive effect of VR based training, except one. Kozak et al. (1993) claimed no transfer occurred from the VR to the real-world task for their selected pick and place task training. However, their finding was debated for the suitability of technology used for the selected criterion task in their study (Psotka 1995).

When it comes to the assessment methodology, among the 17 reviewed studies 5 were user studies where evaluations from the users were the metrics of assessment. The user evaluations generally had positive effects towards VR based training. Except for 2 studies, all other studies used students and volunteers from the universities as study participants. The type of industry ranged from manufacturing, energy, aircraft and automobile maintenance.

\subsection{Firefighter Training}

Effective training is a basis of good disaster preparedness. Among the emergency response occupation domains, firefighter face larger amount of varied environmental threats in their job and require unique skillset to carryout lifesaving tasks (Dunn 2015). VR enables the creation of large and complex training environments that facilitate the training of scenarios which are high resource intensive and difficult to carry out in real life. Due to this, there has been significant attention on VR based training solutions for firefighting (Hsu et al. 2013). 6 studies were identified in the review addressing the 


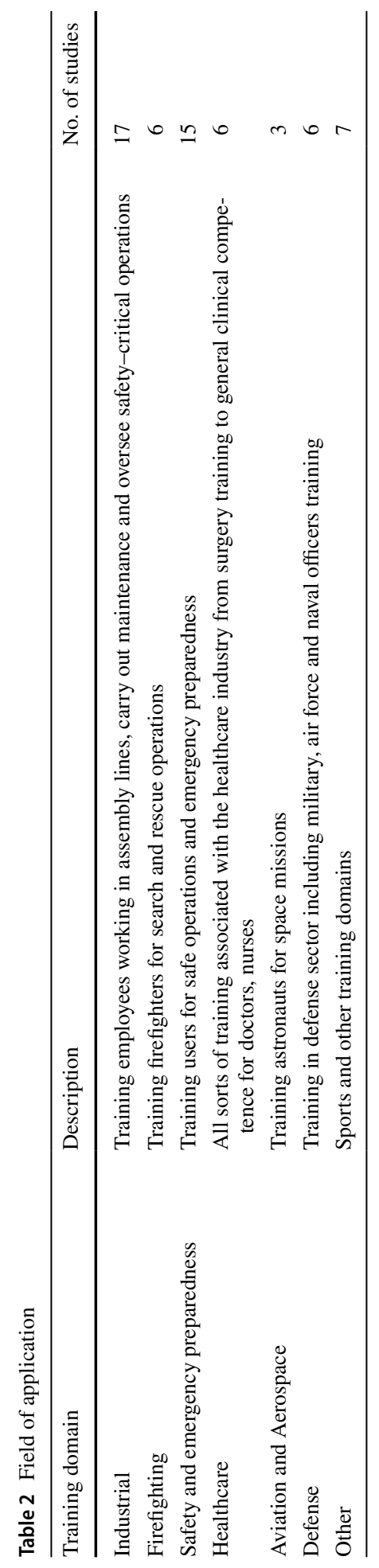




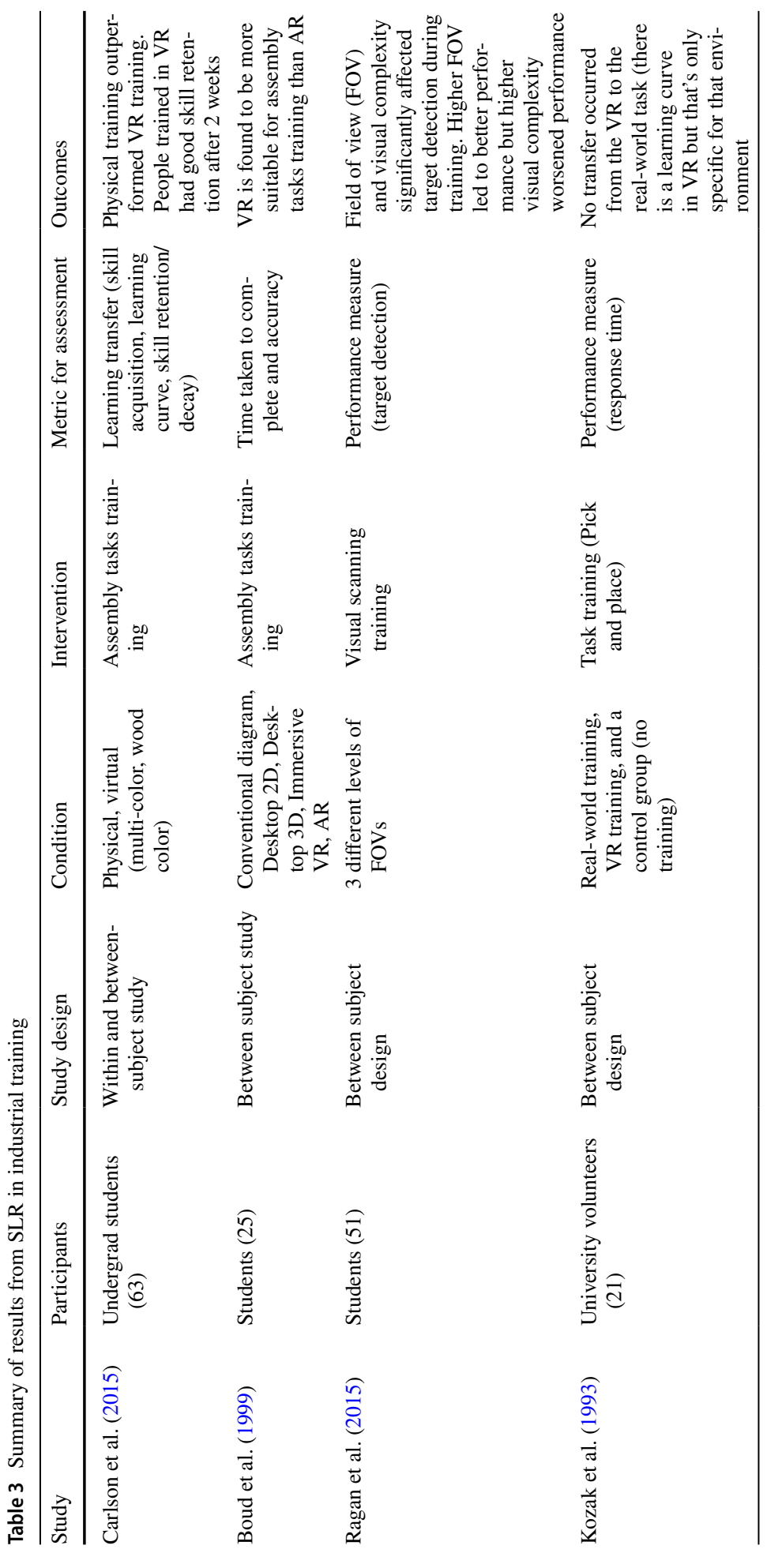




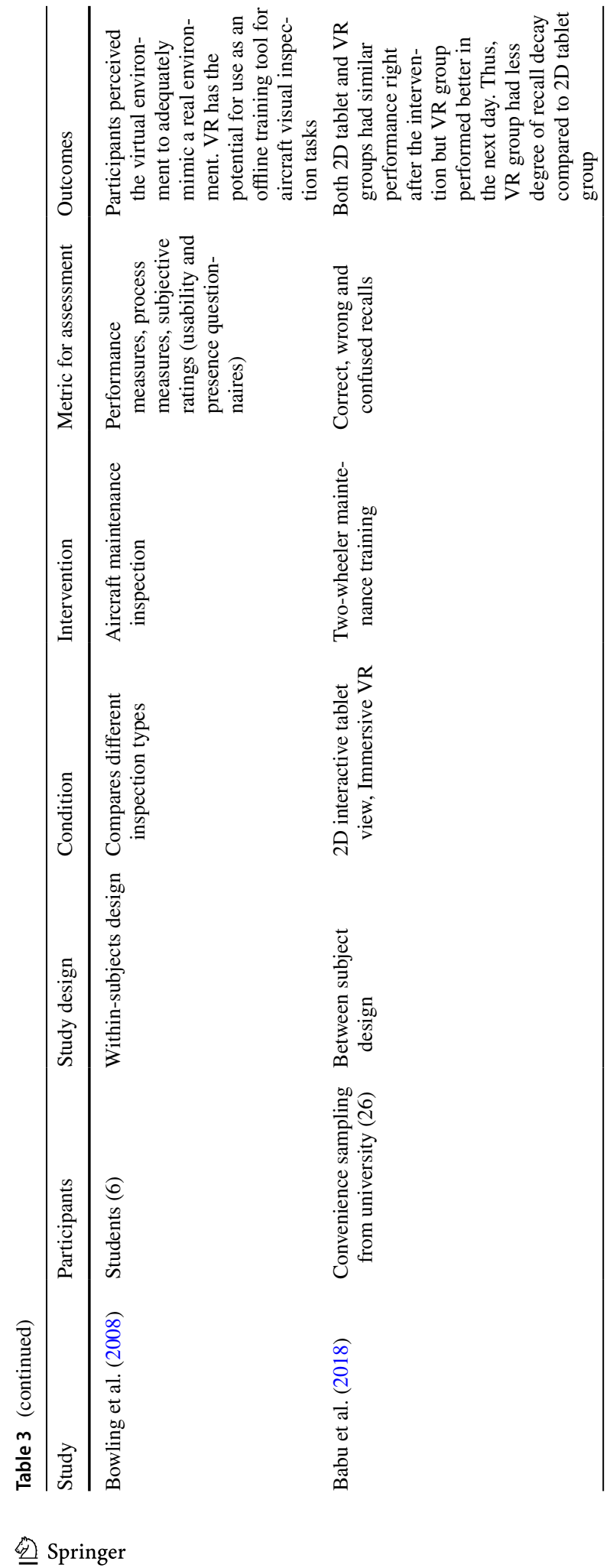




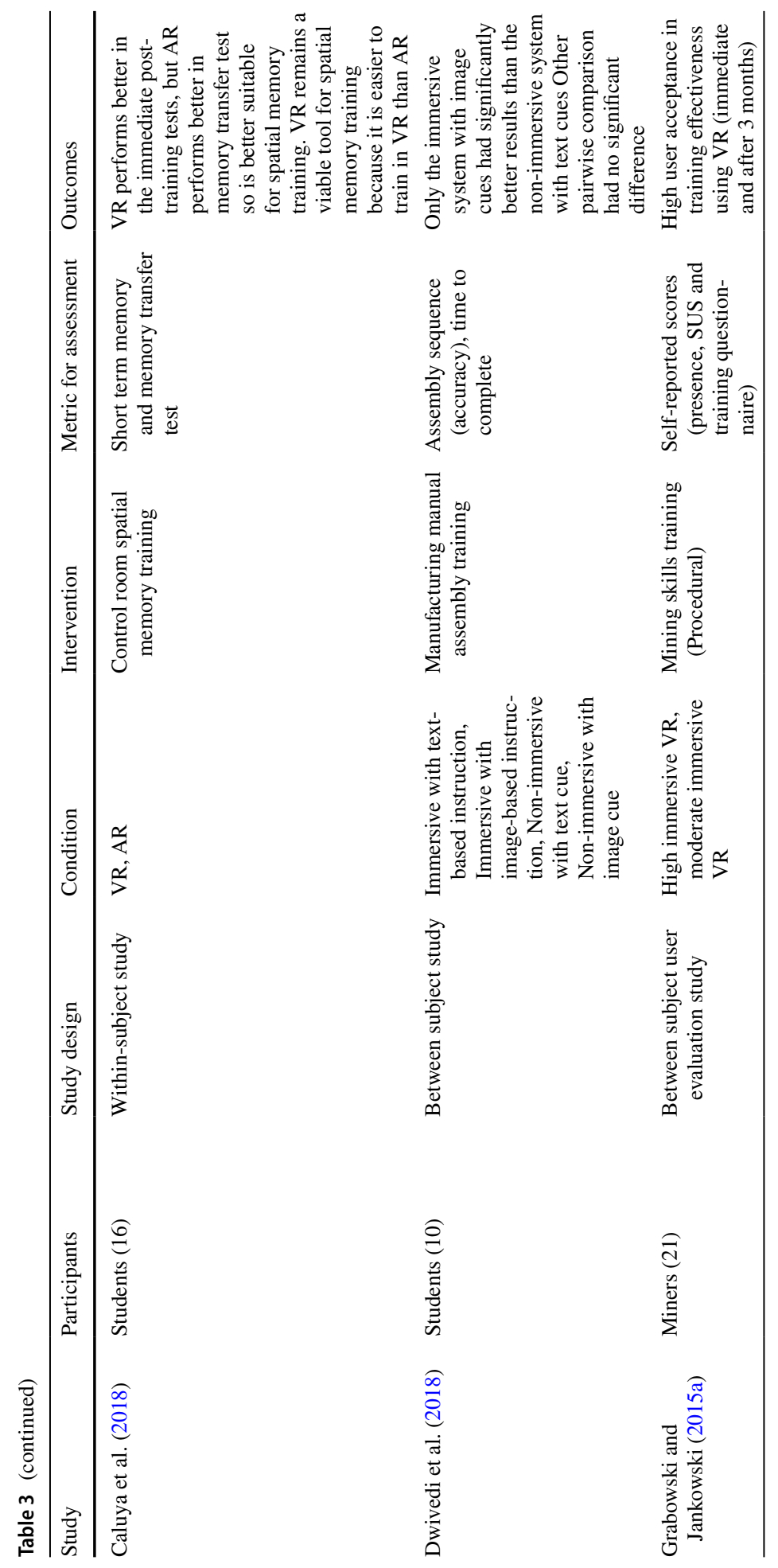




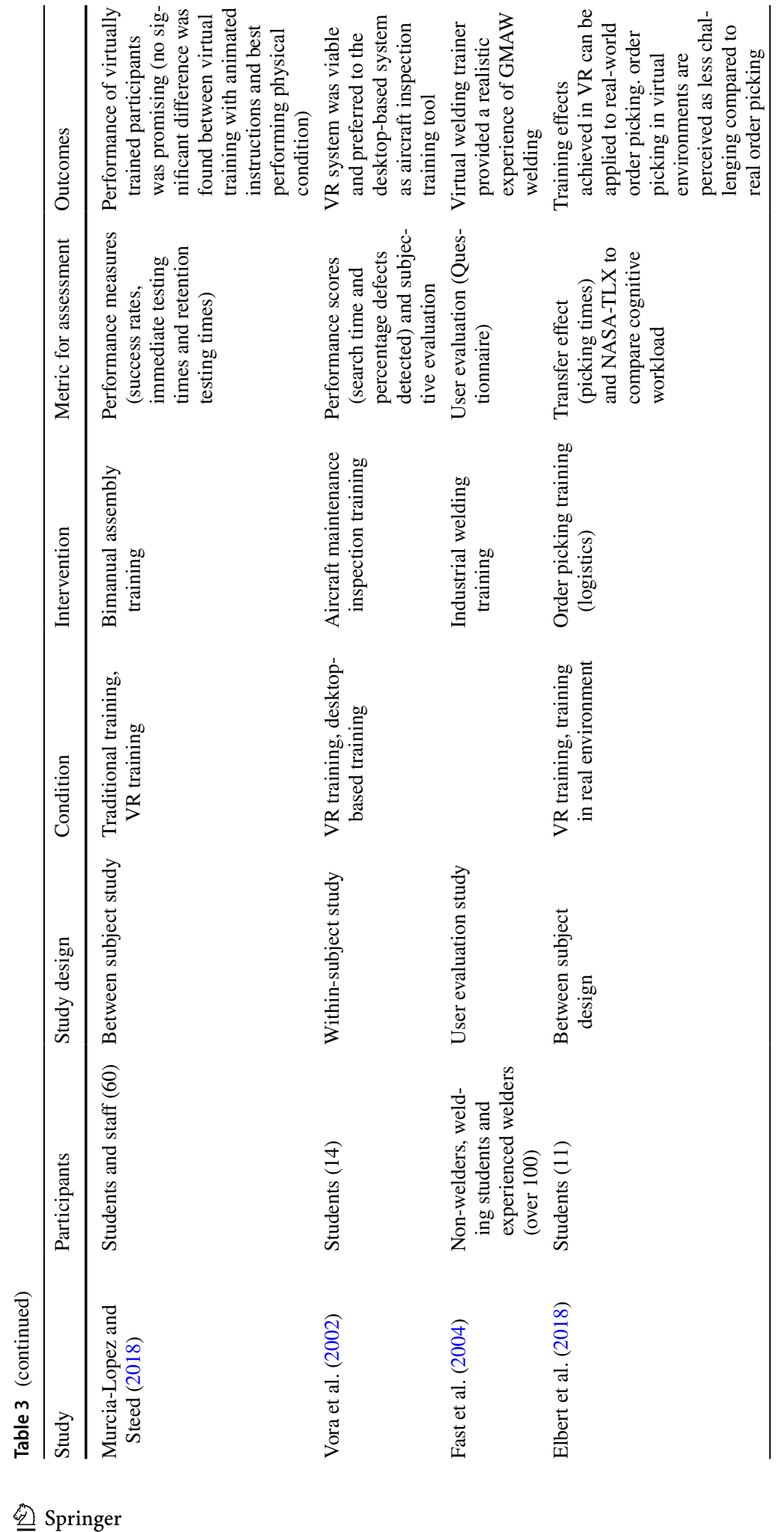




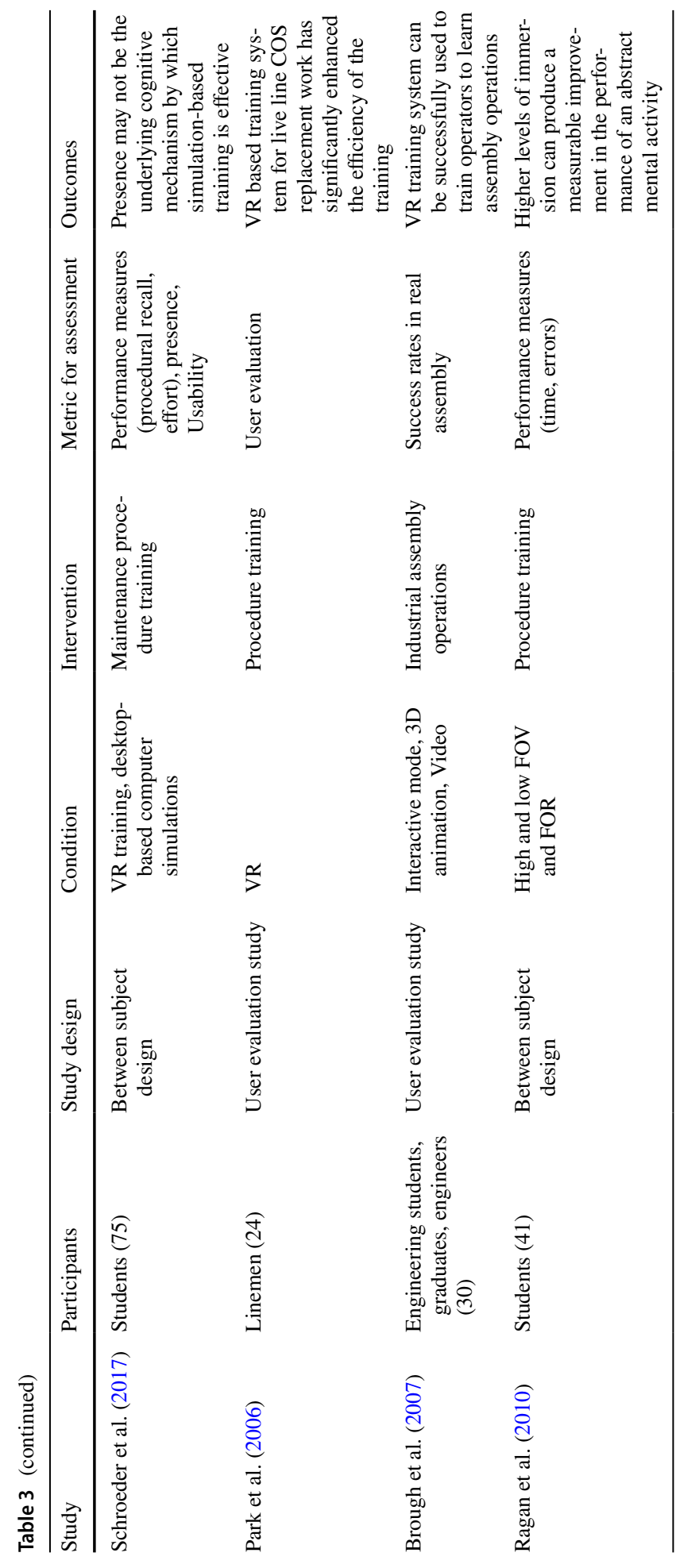


Fig. 3 VR training applications in industrial domain

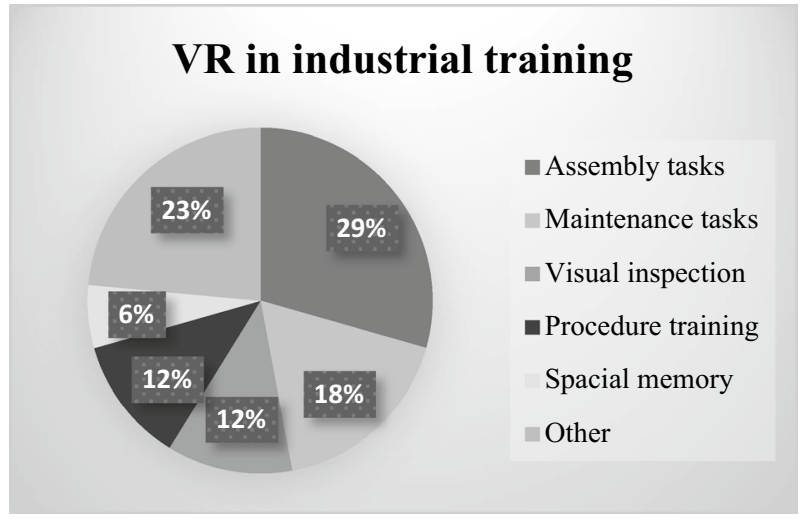

training for firefighters using VR (see Table 4). Firefighters carry out complex spatial navigation tasks for fighting fires inside buildings and rescue operations as part of their jobs. The skills focused in the reviewed studies for training are mainly related to spatial navigation. For firefighting, VR based training is proposed as an alternative, where real-world training is impractical due to cost and personal safety of the trainees.

\subsection{Safety and Emergency Preparedness Training}

Safety and emergency preparedness training is another major application area for VR found in the literature. $25 \%$ of the studies reviewed covers this category of training (see Table 5). When it comes to safety and emergency preparedness training, VR application are seen as a realistic, safe and cost-effective alternative for traditional training methods. For example, VR simulation of emergency preparedness could provide more varied scenarios in a realistic manner and help attain and transfer the safety knowledge to the real-world situations. VR environments in which different disaster scenarios could be simulated provides valuable experiences and training to the personnel for preparing and responding to critical situations ( $\mathrm{Li}$ et al. 2017a, b). This is because trainees are able to learn and practice the skill without risking their well-being and environments.

Because of the realism offered by VR emergency simulations, people show recognition of a dangerous situation in VR and readily produce adaptive responses, making the VR suitable for emergency simulations and for use as an effective training tool (Gamberini et al. 2003). VR- based training produced objectively better spacial knowledge in safety training. When full capability of VR utilized such as active navigation in VR produced better performance (Burigat and Chittaro 2016). Also, VR-based training improved the safety behavior in trainees promising to foster adequate self-evacuation during crisis situations in tunnels (Kinateder et al. 2013).

Almost all reviewed studies proposed VR based training as a novel alternative to traditional modes of training such as real-life drills and table-top exercises for safety \& emergency preparedness. Also the studies reported high levels of engagement among participants in safety training (Buttussi and Chittaro 2017; Haller et al. 1999). 


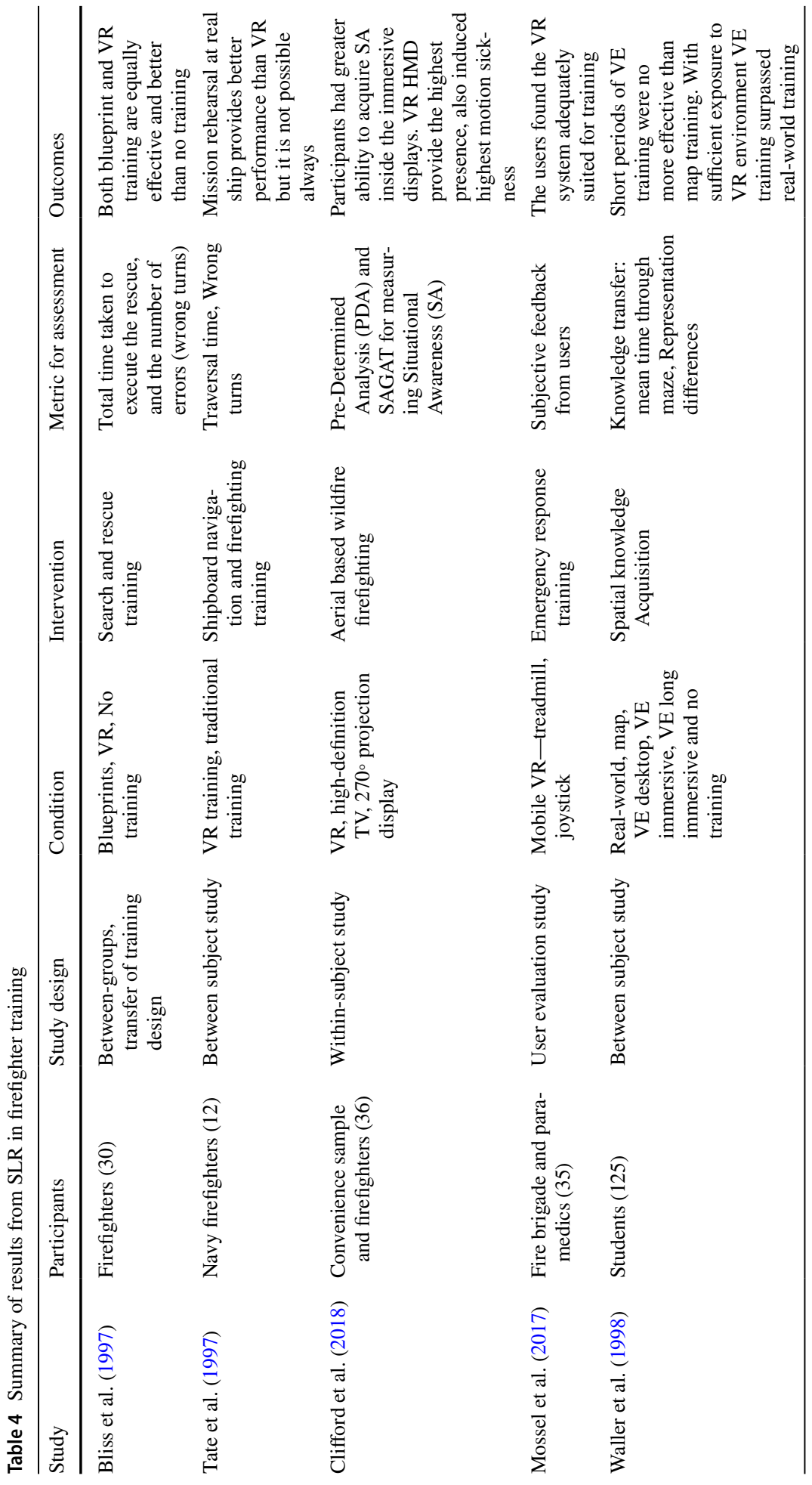




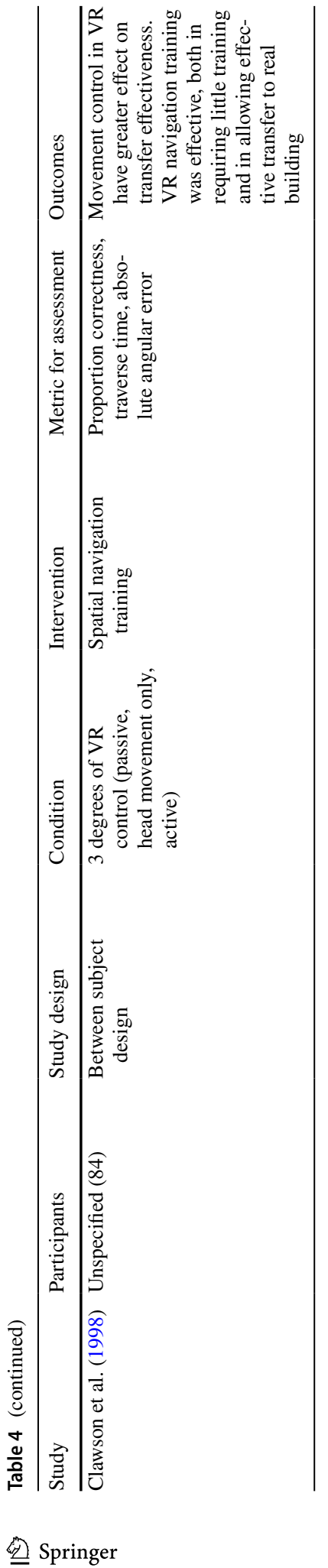




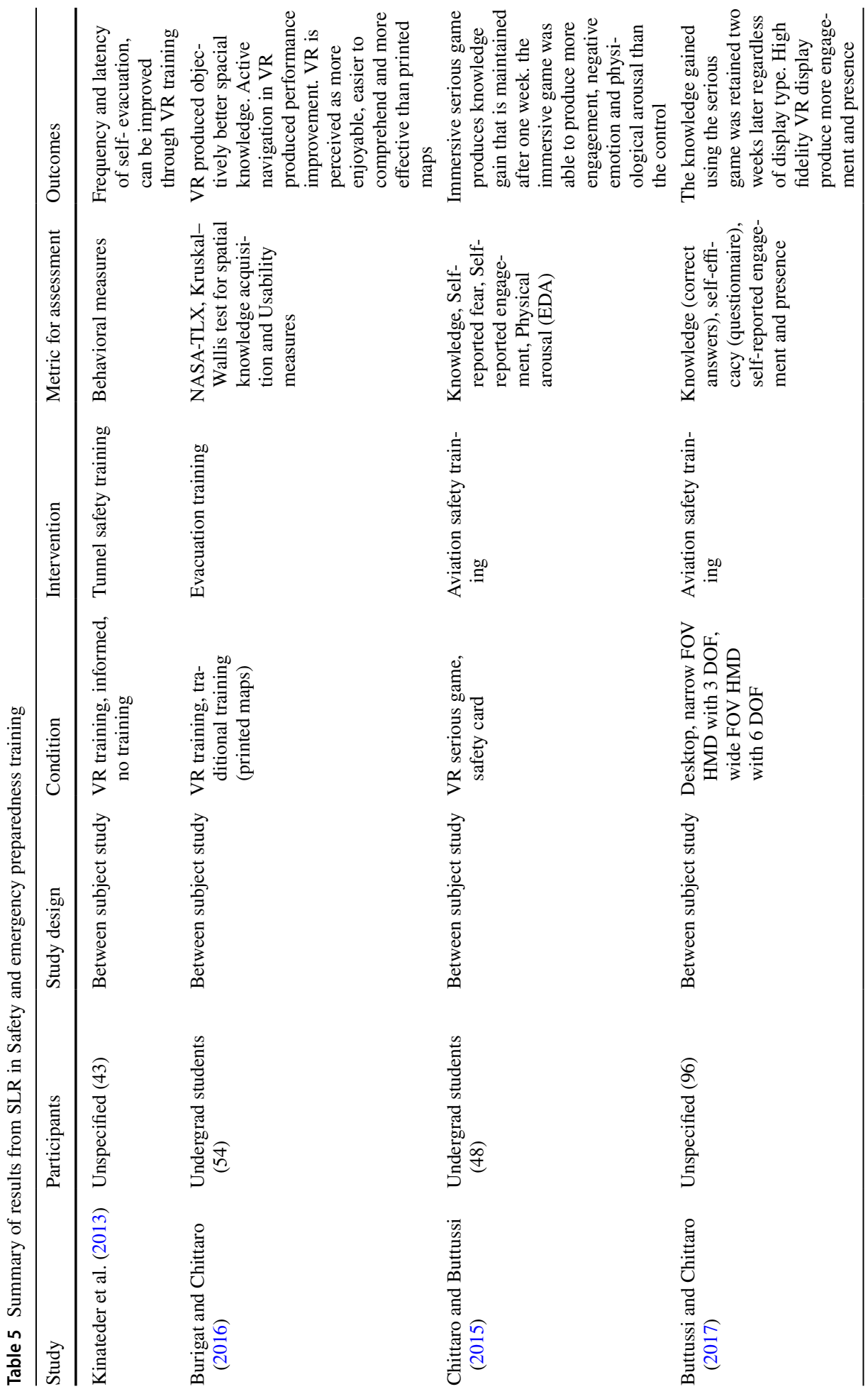




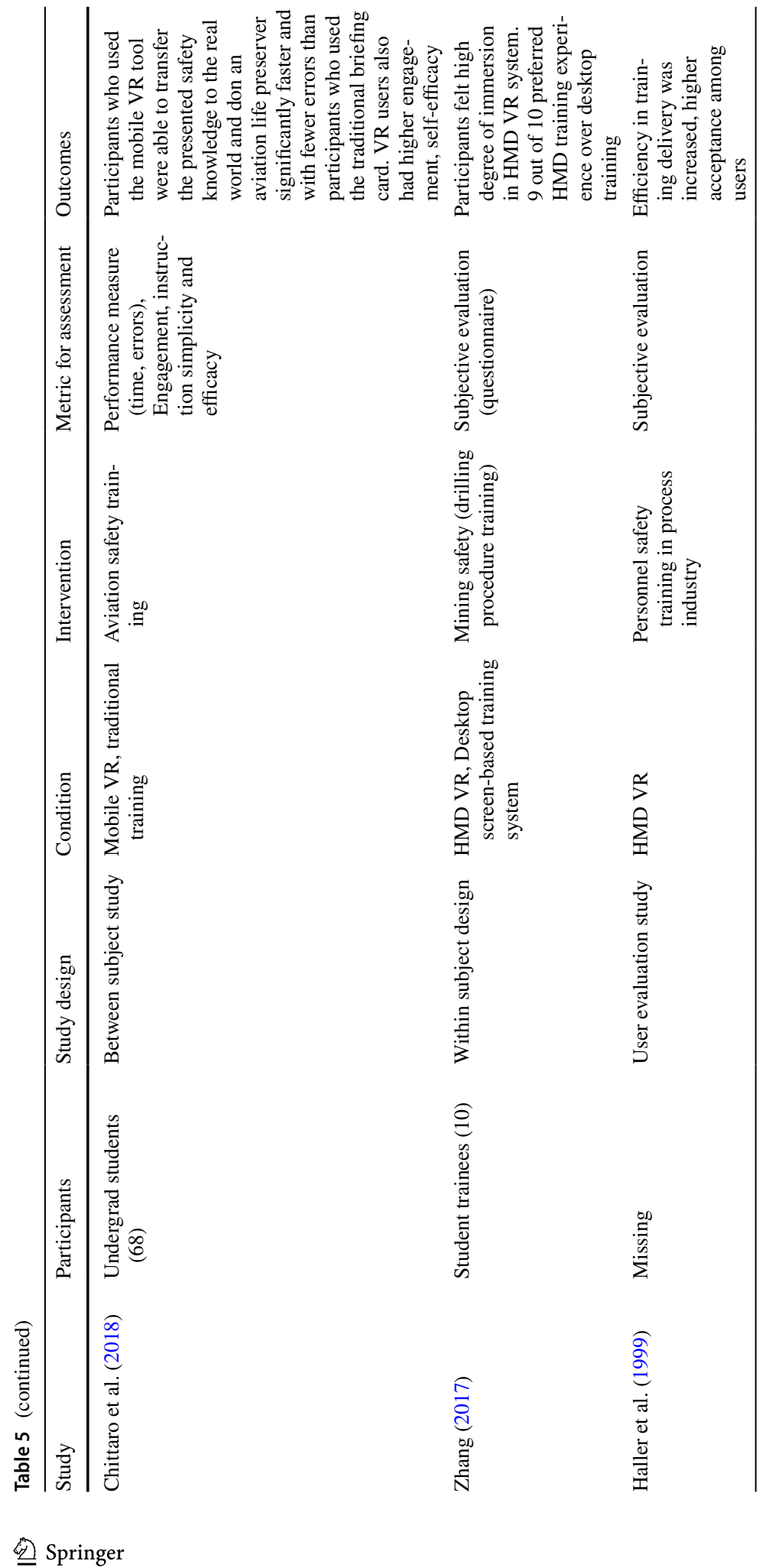




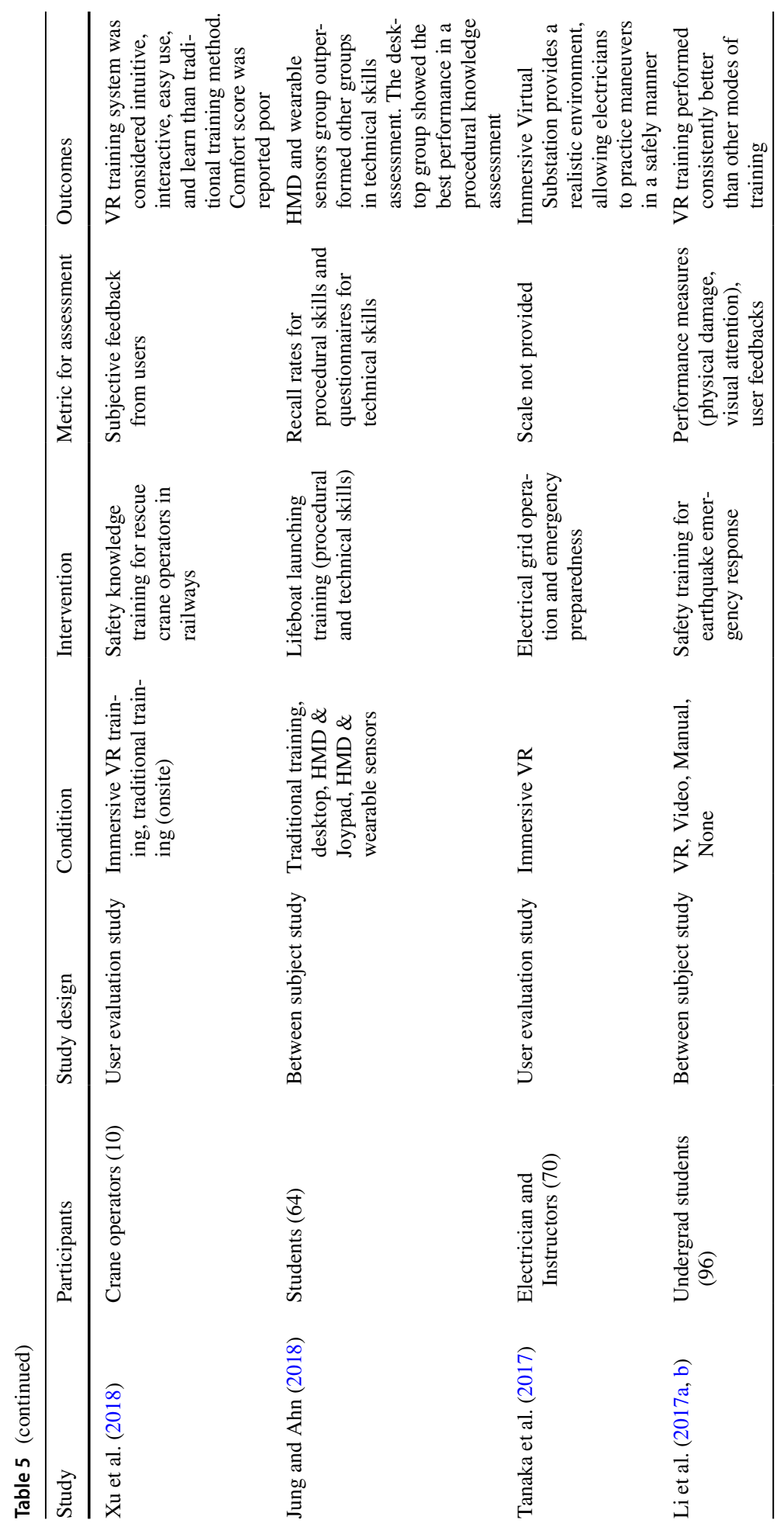




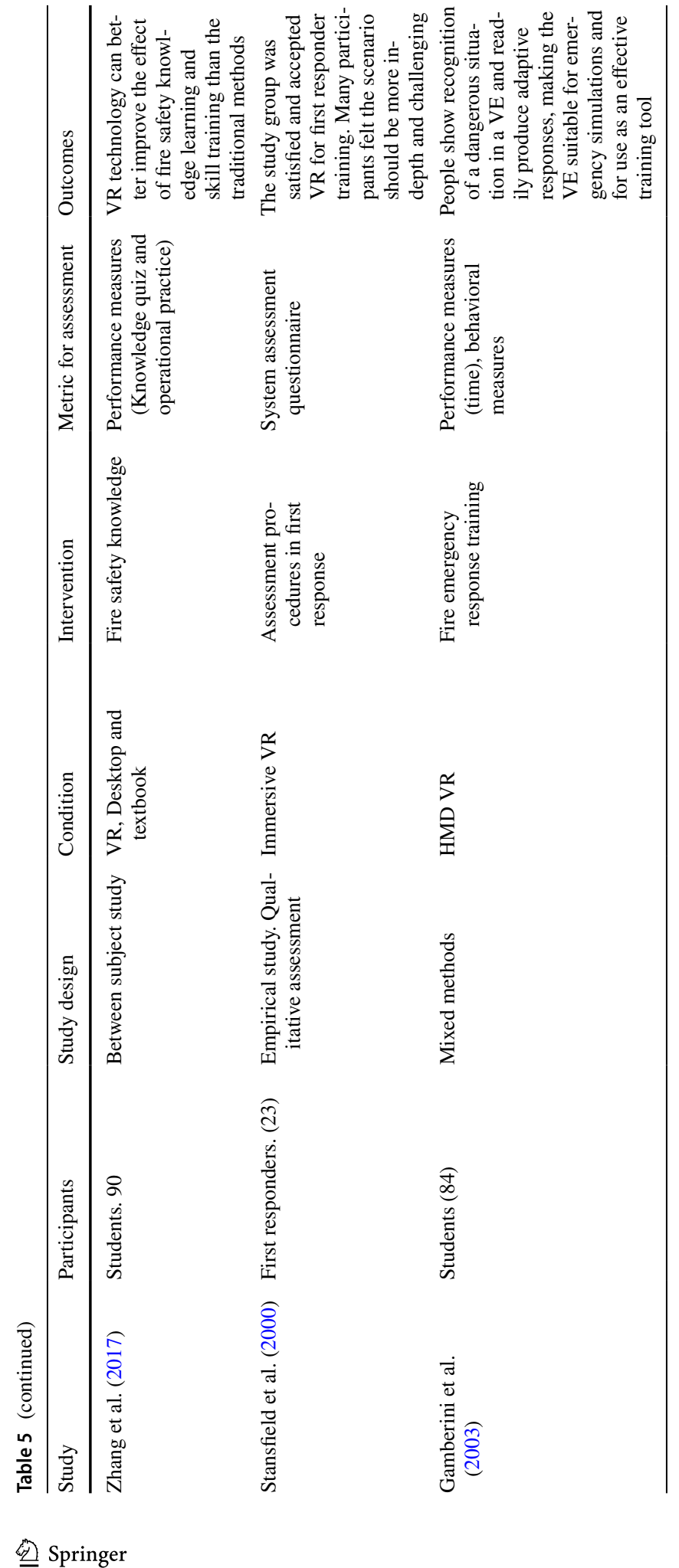




\subsection{Healthcare Training}

The application areas for VR in healthcare are immense and diverse (Ghanbarzadeh et al. 2014). Among the VR training application areas, medical domain has identified and adopted VR for surgical training to a greater success (Gallagher et al. 2005). This is because doctors and dentists are able to train for a variety of surgical scenarios repeatedly in VR without the pressure involved in live surgical procedures. Using simulations for the development and refinement of surgical skills is widely popular in the recent time. With many successful training applications, healthcare appears to be a more mature domain for VR simulation-based training. This is because a wide range of final applications have been studied in the domain. VR simulation applications are found to improve learning outcomes for a variety of surgical procedures (Ahlberg et al. 2007; Bharathan et al. 2013; Grantcharov et al. 2004). However, it is important to note that the VR systems used in surgical training are more sophisticated, have additional special equipment and haptic devices that replicate the feel and behavior of real surgical devices. Healthcare studies that utilized only HMDs are selected for this review (see Table 6) as other VR training systems such as MIST VR trainer are more sophisticated simulators for surgery that falls outside the scope of this review. Although a more mature domain for VR based training, HMD based VR applications aren't predominant in medicine. This could be because surgical procedures trained such as laparoscopic surgery involves camera manipulation, hand-eye coordination and bimanual maneuvering, which are accomplished by observing the monitor and the additional affordances of HMDs don't add extra value here ( $\mathrm{Li}$ et al. 2017a, b). Huber et al. (2017) confirmed it in their study where combination of LapSim (from Surgical Science, Sweden) with HMD led to high levels of immersion, but there was no significant difference in the training effect. However, there are some use cases in healthcare training VR HMD's seem to add value, such clinical competence training for nurses (Dang et al. 2018) and operating room fire prevention and control training (Sankaranarayanan et al. 2018).

The skill trained in the health care domain range from cognitive skills such as surgical procedure (Yoganathan et al. 2018), safety procedure in operating room (Sankaranarayanan et al. 2018) and motor skills related to surgery (Anglin et al. 2017).

\subsection{Aerospace and Aviation Training}

Training in contexts that are impossible or very difficult to create or simulate is a reason for using VR in space training. Training for space operations has inherent challenges due to the very limited chance for on-the-job/hands-on training possibilities. There have been some successful use cases for VR based training systems in the space and aviation domain. One such use case is the Hubble scope maintenance mission training in VR. In the pebble repair mission, the astronauts had to operate a tele-robot in space using five different camera views on three monitor. VR-based training was utilised for enabling the astronauts to develop and maintain a dynamic 3D image in their head about the manipulator system to the Space Station, Shuttle and Payload (Logan 1998). For the space shuttle mission training, limited availability of personnel and training facilities made it challenging for training a large number of flight team members. The training facilities and simulations are prioritised for the crew and selected members of the primary flight team. Hence, the support and planning teams get very limited available time for training. An effective training was required for a 100-person team without the possibility of using the real telescope. VR was adopted for solving this challenge and to provide accurate knowledge of the geometry of 


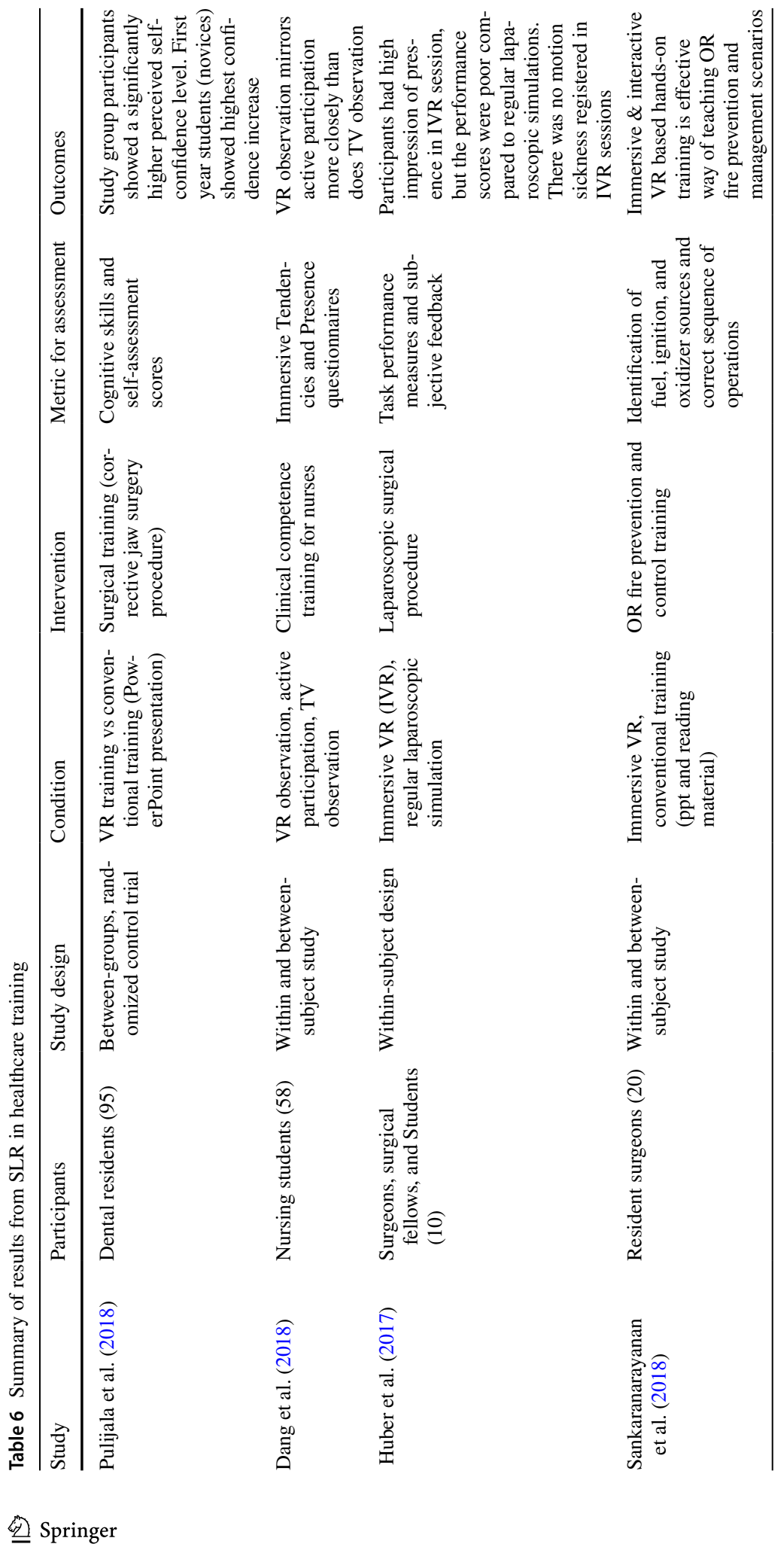




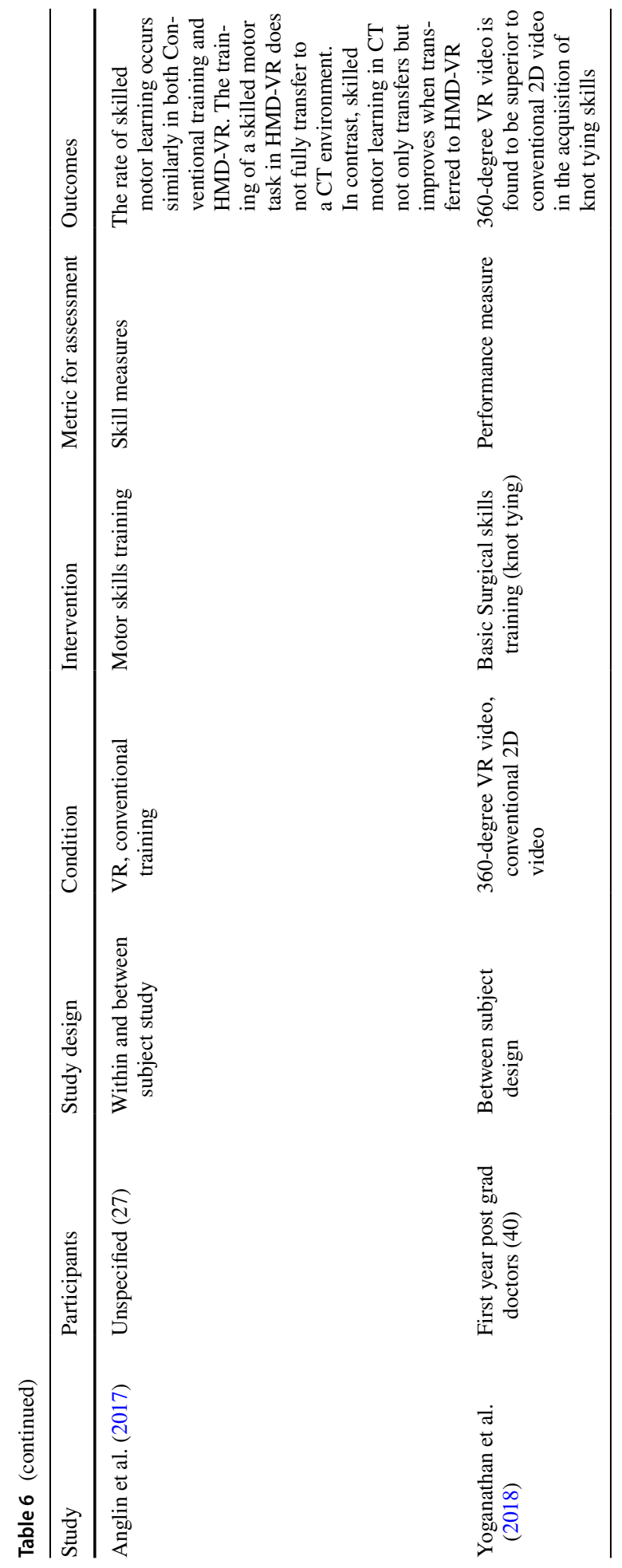


Hubble Telescope and procedural steps of the planned maintenance (Loftin and Kenney 1995). It was also noted that this VR-based training design was supported both by a task analysis method for training requirements and an intelligent tutoring system was utilised to define the learning scenarios.

VR was used for training both cognitive skills associated with task procedures and psychomotor skill associated with spatial orientation in the aerospace domain. Table 7 summarizes the results for these studies.

\subsection{Defense Training}

Military has long relied upon simulation-based training for preparing and keeping the soldiers battle ready. VR has been adopted in military for training due to its ability to allow defense personnel to try a range of simulations, without the associated costs, thus highly reducing training budgets while increasing safety. VR is applied in defense training programs, with simulators ranging from basic shooting systems into more immersive situations. VR enable the trainees to experience a particular situation within a controlled environment. For example, a battlefield scenario in which they can interact with events but without any personal danger to themselves. Soldiers can train in any number of different situations, environments and prepare them for combat situations or other dangerous settings. In the reviewed studies VR is mainly used in military training for providing situational experience such walking through the trainees in extreme situations such as parachute jump, navigating in the jungles, behind the enemy lines etc. For example, in the study investigating VR for topographical training, soldiers trained with high level VR showed better directional accuracy than other training modes (Singer et al. 1997). In another study on aerial door gunnery training, VR group outperformed LCD display group in gunner performance (Stevens and Kincaid 2015). Four of the reviewed studies were from the late $90 \mathrm{~s}$ that used previous generation VR technology (See Table 8) which could partially explain the interaction challenges by the participants in some of these studies from that period (Hall et al. 1998). Research related to defense usually takes longer period to declassify and normally not published in the research articles. This could be a reason why we haven't found many military VR training application in our search. The summary of the results from the VR applications for defense training is provided at Table 8.

\subsection{Other Domains}

Apart from the above-mentioned 6 major application domains, there have been attempts to utilize VR technology for training for sports, teacher training, calligraphy, etc. VR has been found to improve the awareness of the kindergarten caregivers to the toddlers' emotional experiences (Passig and Noyman 2001). VR assisted teacher training also found to significantly improved Classroom Management skills of Pre-service teachers (Lugrin et al. 2018). In the study focusing on oriental calligraphy, it was found that that training and transfer effects of VR were comparable and, in some situations, better than real training (Yang and Kim 2002). There are also increased interest in sport training applications for VR due to the realistic experiences' athletes might get from VR simulations. In the study investigated American football training using VR found two significant benefits of using VR for training: (1). VR provides trainees the ability to continue practicing their mental preparedness, (2) VR effectively and proactively reduces the injury possibilities of trainees by avoiding the physical impacts particularly to the player's heads (Huang et al. 2015a). The results from these domains are summarized in Table 9. 


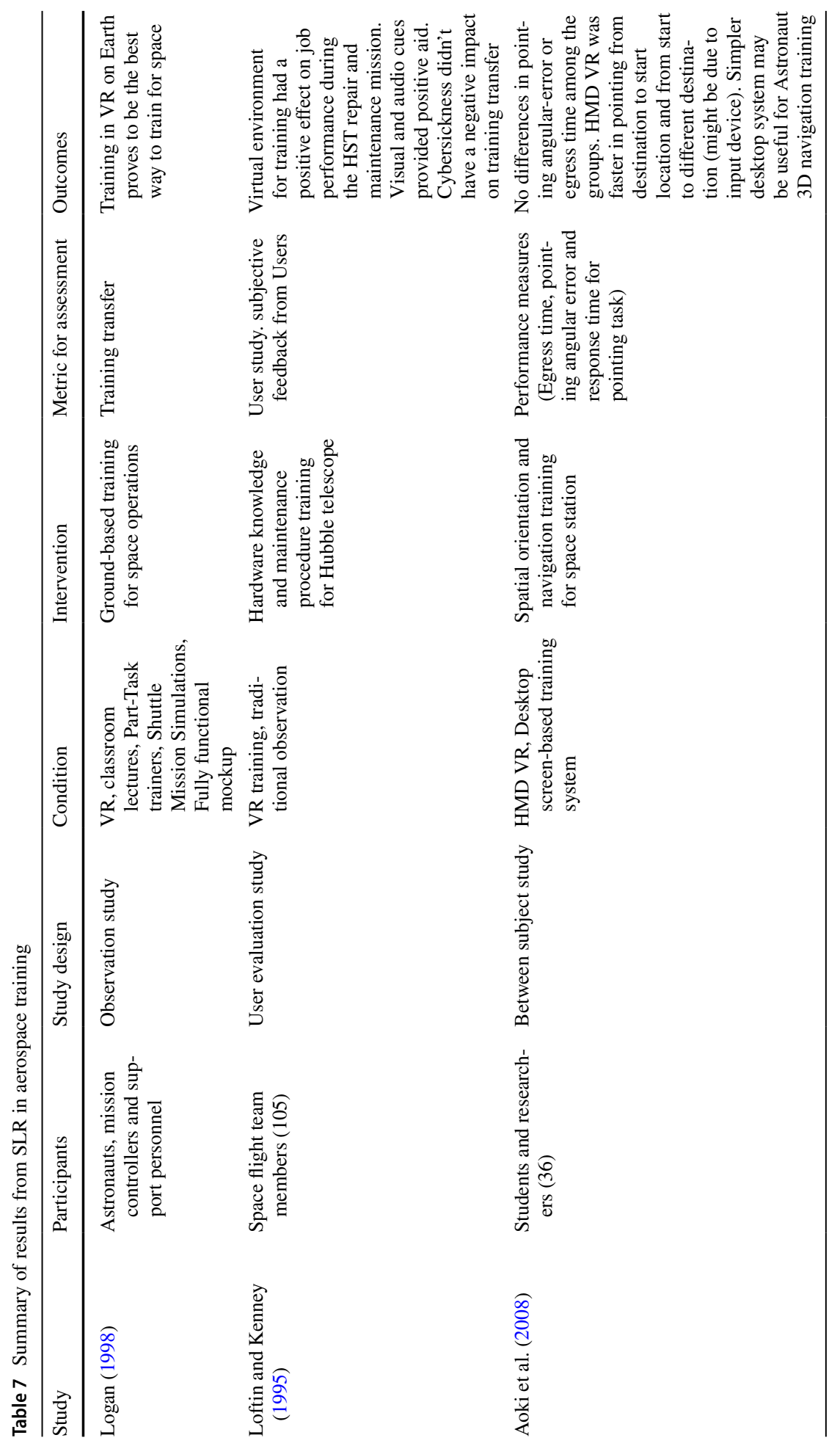




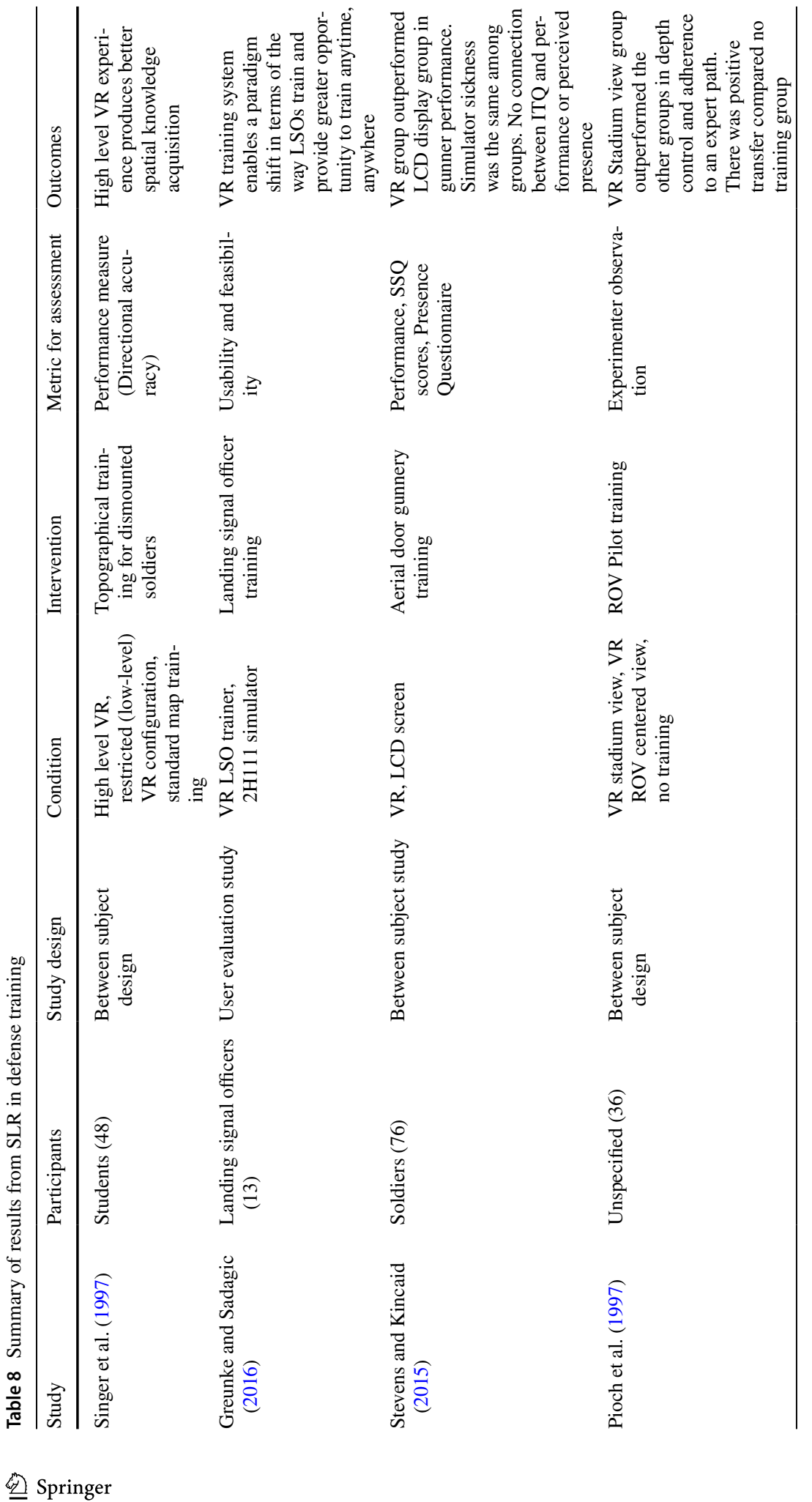




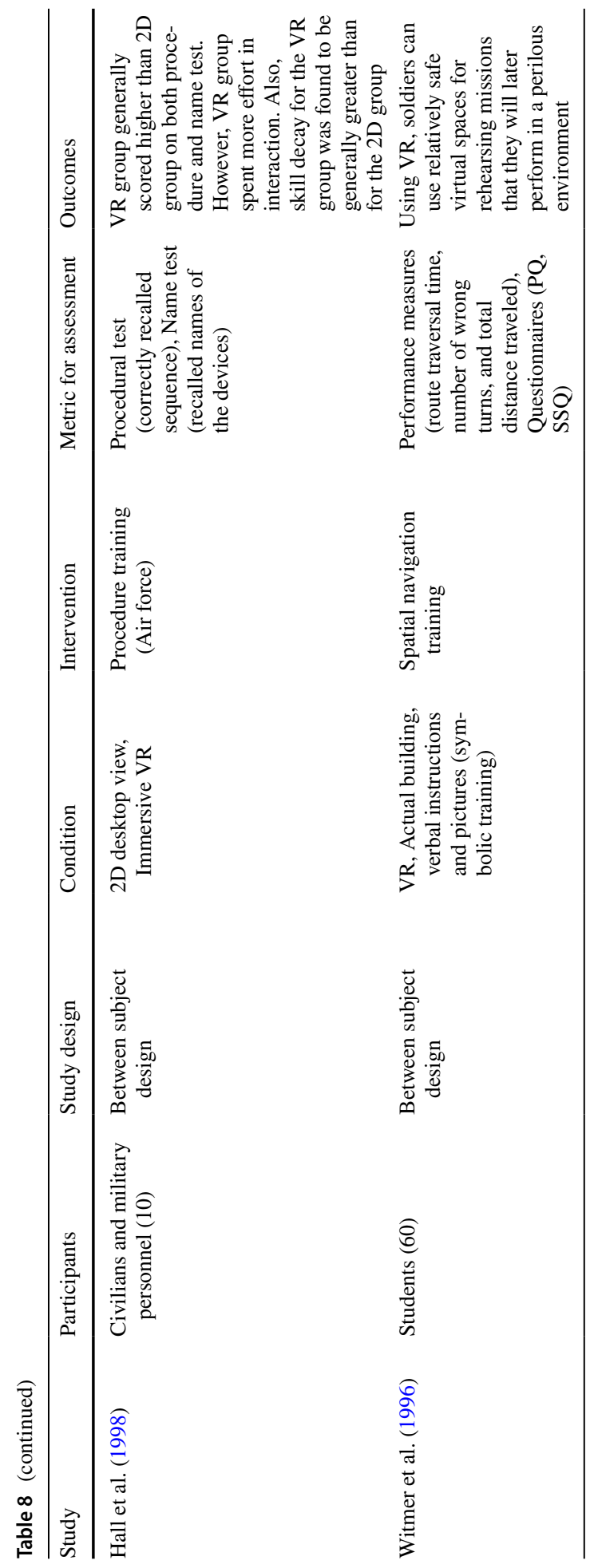




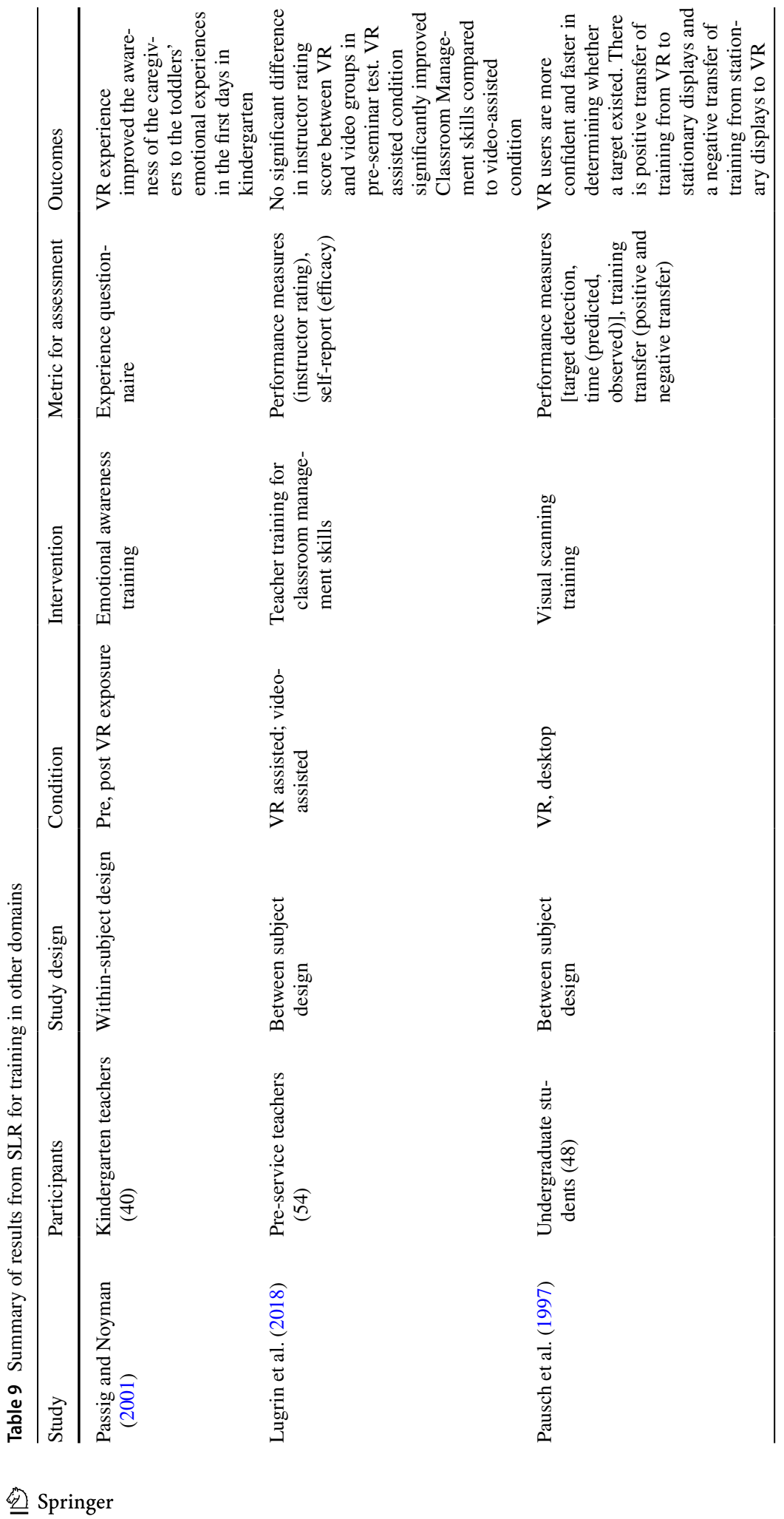




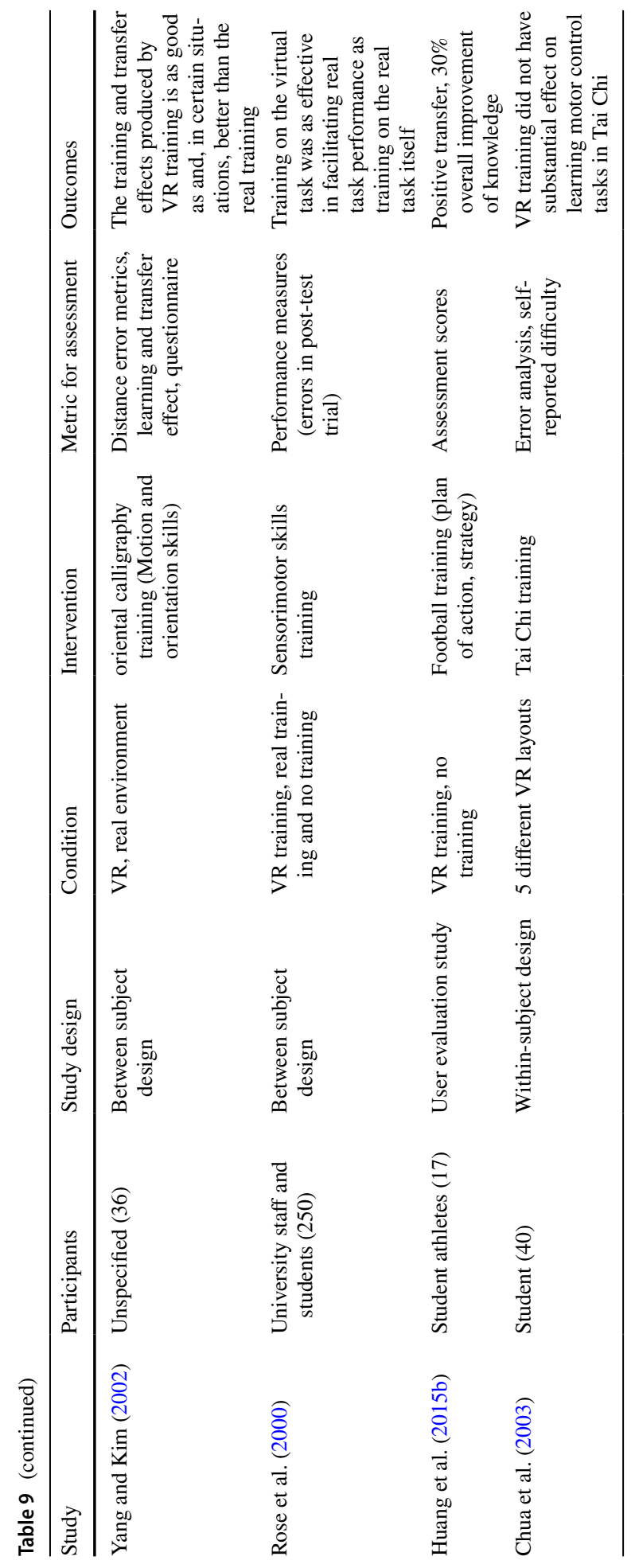




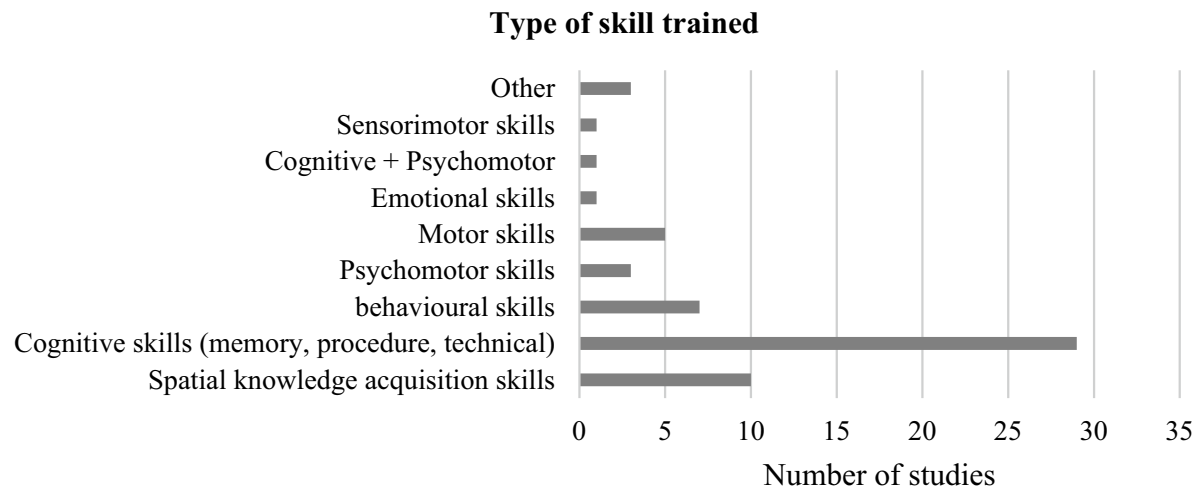

Fig. 4 Type of skill trained using VR

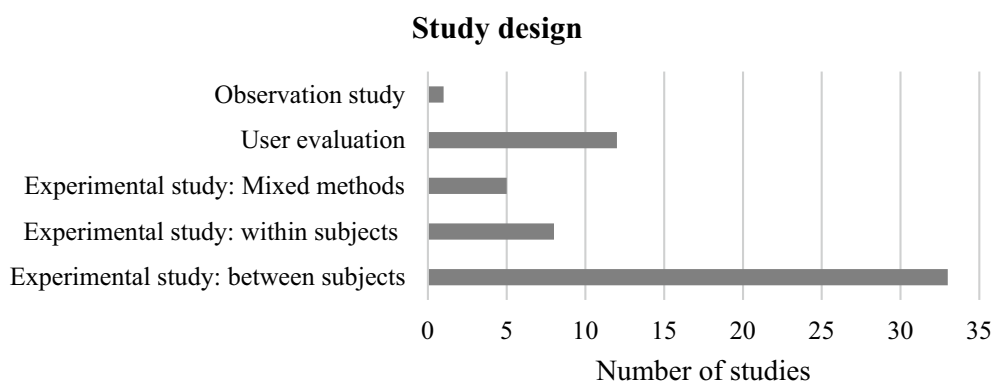

Fig. 5 Experimental method used in the studies

\subsection{Summary of Types of Skills Trained}

Figure 4 shows the breakdown of the types of skills trained in the review studies. Skills related to remembering and recalling procedures are most trained skills in the reviewed articles. This is in line with the high numbers of assembly, maintenance and procedure training applications. It is followed by spatial knowledge acquisition skills such as visual scanning, head movements and observation skills. VR has an advantage for training spatial skills due to head tracking (more natural interaction) and immersion. There are also studies that investigate VR's ability to train skills such as behavioral, emotional, motor and sensorimotor skills.

\subsection{Training Evaluation Method}

When it comes to the methodologies for assessing the effectiveness of VR based training, evaluating the task performance post training is the extensively used method. 33 studies used between subject design for their experiments, 8 studies used within subject design and 5 used mixed methods as their study design. The metric for assessment comes under 3 main categories: (1) Performance measures; (2) Self-reported measures and (3) Observations. 
Performance measures evaluate the acquired skills and knowledge post exposure to the VR based training. The reviewed studies used performance measures such task completion rate, accuracy, sequence of steps done right, error rate, temporal, and behavioral measures etc. 12 of the reviewed studies were user evaluations. These are mainly self-reported measures such as questionnaires, rating scales and interviews that capture the user perceptions on the training methods. User perceptions included self-efficacy score, user satisfaction, usability, self-reported difficulty, immersion and presence scales, etc. Observations are the expert evaluations and researcher's survey about outcome of training. Figure 5 shows the breakdown of study designs among the review articles.

\subsection{Types of Study Participant}

The type and number of study participants add remarkable weight to the significance of conclusions and generalizability of each study results. University students and staff are the most commonly used participants in the review studies (See Fig. 6). Although the VR training applications were meant for professional training, only 19 of the reviewed studies used industry professionals and 6 studies used professional students as study participants. Rest of the studies appears to be utilizing convenience sampling since majority of the VR training applications developed and tested in university laboratories. This sampling bias raises the issue of how well the target populations are represented in these studies.

\subsection{VR Proliferation}

The publication years of the reviewed articles follow the technology trend of VR as discussed in the background section. As expected, there is a clear increase of research publication in the period after 2013. 52\% of the reviewed article were published in the period between 2013 and 2018 (Fig. 7). This is in line with the proliferation and popularity of the technology post the arrival of HMDs that are both more efficient and affordable. Compared to the VR studies from the $90 \mathrm{~s}$, VR technology has improved to an impressive degree. Especially improvements in computing power and display technology have solved many of the problems from the previous generation of VR in the $90 \mathrm{~s}$, such as low resolution, high latency, heavier headsets, etc.

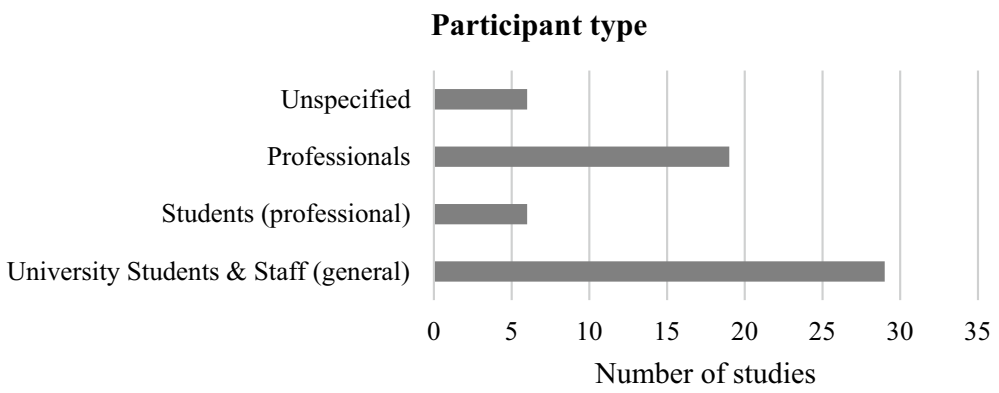

Fig. 6 Types of study participants 


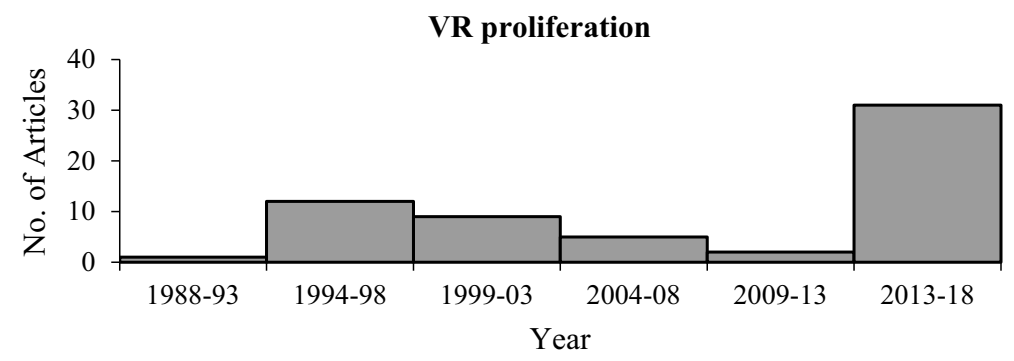

Fig. 7 VR proliferation in training research (temporal evolution of publications)

\section{Discussion}

This systematic literature review retrieved a range of VR applications that have been developed for training across various professional fields. Our search identified 6 major domains of VR applications in training in the literature to date, designed to train people in professional settings and for specific job-related competencies. In addition, few emerging application areas, such as sports training, teacher training etc. were identified in the review. VR applications are innovative tools that promises to have huge potential for education and training in general. The key benefits of VR mentioned in the reviewed studies are, VR allows trainees to understand the spatial relationships and concepts, and provides meaningful, contextual and situated learning experiences. In the applications listed in the review, VR helps to create realistic simulated experiences. Several of these VR applications were viewed and argued as a valid and reliable method for training. The studies that spanned over 30 years used different VR devices based on the stateof-the-art of that time. These devices ranged from very low resolution-high latency to high resolution-low latency, varying quality of tracking and locomotion mechanisms. Although technology limitations affected the user experiences, key features of VR such as high immersion and presences felt by the users have generally led to better performances in different learning situations. Studies carried out in space training concluded VR to be the most effective training medium for space operations (Loftin and Kenney 1995; Logan 1998). However, in this particular use case the VR training application was supported by a thorough job analysis and smart instructional design methods (Mellet-d'Huart 2009). Training in VR is shown to increase trainees' confidence (Pulijala et al. 2018), subjective attractiveness (Zhang 2017), enhancing skill retention (Babu et al. 2018; Carlson et al. 2015) and performance (Hall et al. 1998; Jung and Ahn 2018). Learner engagement and motivation is particularly important in safety training because more engaging and experiential safety training were found to be more effective than traditional training methods (Burke et al. 2006). The advantages of VR-based training are well-established by now. VR facilitates safe training environments, supports rehearsal of responses for emergency situations, enables skill acquisition through learning by doing, and provide training opportunities where training in real situation is not possible due to safety risk, cost and practicality. VR-based training could also be useful where real life or other training modalities are feasible due to the flexibility of VR for repetition, providing feedback and adaptive learning possibilities (Vaughan et al. 2016). Nevertheless, there are also experimental results that showed conflicting evidence on VR benefits (Kozak et al. 1993) or no benefits at all (Chua et al. 2003; Huber et al. 2017). In many of 
successful VR training instances, it is important to note that the simulation and its accuracy in connection to the training objective is more important than the VR technology itself. Surgical training is a good example for this where VR based training is successful still high immersive VR HMDs aren't generally preferred. In the following section, we discuss the identified challenges for VR-based training and come up with recommendation for VR adaptation for training in professional domains.

\subsection{Challenges for VR in Training}

There is a clear indication from the review for the increased interest in applying VR in professional training in recent years. Although VR was used for specific training situations for many decades, the technology is still fresh for most domains. To date, the education and training carried out through simulators is predominantly driven by technology and cost (Neubauer et al. 2017; Stedmon and Stone 2001). Simulator developers decide upon the specifications of consumer electronic technology based on their specific business model (e.g. technology level vs. cost), and ultimately what customers are willing to pay for. The details and sophistication of the simulators are directly connected to the cost of the simulator. The recent interest on the HMDs and their application into training were based on the fact that the hardware is now much more advanced, relatively cheaper and facilitate highly immersive simulations that were only possible in high-end expensive simulators previously, if at all. As the simulated environment in VR is fully digital, it is also believed to be much more flexible. However, there are a few important limitations that need to be paid attention to. The first main limitation is the simulation content for VR. Production of VR simulations is still very expensive, and time consuming. Most simulation applications tested in the reviewed studies were developed in laboratories for very specific contexts of use. How this translates to real-life training setting with varying training goals is still unclear. In addition, VR is still relatively a new type of Human Computer Interface (HCI) where the users are immersed and interact in 3D. Formal understanding and evaluation of interaction within VR is problematic because of the limited understanding of HCI in immersive 3D (Bowman et al. 1998). Due to the immaturity of interaction design techniques for VR simulations, the user experiences vary to a large degree between different simulation applications. User familiarity to the technology is an important challenge to address. In the review, it was identified that several studies reported cases of cybersickness and users struggling with the interaction in VR simulations.

The second main limitation lies in the VR hardware. Current consumer-grade VR HMDs are primarily designed for entertainment and media consumption. They were not designed keeping prolonged usage for educational and training settings in mind. VR device manufacturers themselves recommend frequent breaks while using VR HMDs to avoid discomfort. In traditional simulators, students could train for hours without break, but this is not possible yet with the current VR HMDs. The existing curricula for training might need to be modified for adopting VR HMDs.

In addition, there are also potential limitations, such as safety of the users and usability issues while adopting VR for training. Other personal factors such as disabilities, eye issues, etc. could also influence the utilisation of VR for training for a wider range of users. In an educational and training context, this is critical because the educational and training medium should be usable by most of the target population. 


\subsection{Recommendations for VR Adaptation in Training}

Based on the review results on lessons learned and challenges for VR in training the following recommendations are proposed. Firstly, pedagogical and interaction aspects between the users and VR application should be carefully considered while adapting for training. In order to achieve a high degree of effectiveness, the design approach for VR based training should be supported by thorough analysis of the task and good instructional design methods. The training outcome and skill proficiency criteria should be well defined in order to assist trainees to develop skills through VR-based training.

When it comes to training applications for VR, the underlying learning theories are often overlooked or not paid enough attention. Most of the reviewed studies did not explicitly mention or explain the learning theories the VR applications were based on. Learning theories such as constructivism and experiential learning were briefly mentioned but how these theories are utilised in the development of their applications weren't explained. This makes it challenging for the generalisability of the findings from the studies. In other instances, especially the studies that had user evaluations as assessment methodology mainly focussed on the usability of the VR application rather than the actual learning outcome itself. This we suspect is mainly due to the novelty of the technology to the particular domain and researchers were merely playing with the technology and ideas of applications. So, the future VR training applications should have clear training objectives and goals training outcome. Also, the training outcome should be evaluated through robust assessment methodologies to objectively measure the trainee' increase of knowledge and skills in addition to measuring their training experiences.

Many of the technical challenges faced by the earlier studies, such as poor resolution, high latency and tracking issues are resolved in the latest generation of equipment. VR technology has been heavily updated in recent time and it renders many of the usability issues, quality of experience and cybersickness results from older results invalid or less likely to occur with the new technology. However, these human factor issues are non-negligible in training applications. Once incorporated, the technology will be used by people in various age groups, gender and technology familiarity. With the different stakeholders and multidimensional nature of learning process combined with the complexity of VR technology, simulator developers and researchers should focus on the usability. It is important to develop and validate methods to quantify the quality of experience in VR, so that the impact of VR training applications on the users could be measured and better training experiences created. It was also evident from the review that there are varying maturity levels of VR applications between domains, and still a lot to be done for the development of VR applications for training. Also developing and sharing best practices for VR training applications within and across domains might prove useful for avoiding common issues such as familiarization and usability challenges.

Finally, it could be safely said that VR has survived the initial phase in technology adaptation and has shown good potential as a training tool for many professional domains. However, before VR applications can be widely used as a training medium it must be demonstrated that it is an effective medium for learning skills and transferring them to real life settings. It is important to thoroughly investigate VR's applications and assess its true value and place within training before implementation. 


\subsection{Future Research}

To better understand the barriers in VR based training and find ways to reduce them, the future research should move away from laboratory-style experiments and focus on the use of educational VR in realistic settings, as part of educational or training programme. As presented in the findings above, the actual context of use has a great influence on the learner experience. Majority of the reviewed studies focussed on very short-term use of VR-based training tools in experimental settings. However, we couldn't find many followup studies on the retention of skills acquired through these innovative training methods. Future research should also examine the effects of prolonged and repeated usage of the technology in skill acquisition as well as the user's motivation on using the technology.

Since VR training applications are heavily reliant on technology, educators and researchers have to rely on the technology developments from elsewhere. The development of VR technology is predominantly driven by industries outside education such as gaming and entertainment. The generalisation of VR technology for training is a key issue to address in the future research. Apart from few bespoke VR HMD's that are tailor made specific training needs, majority of training application would have to settle with off-theshelf VR technology. The field could benefit highly with a guideline or blueprint to select the right VR technology for its application requirement.

The future studies should also be explicit on the theoretical foundations of their VR application and describe the development process of their training and course content.

One main reason for the renewed interest in VR technology is the cost and availability of the hardware. However, the cost-effectiveness of VR simulators for training compared to other training approaches is still largely unknown. Although the hardware cost has dramatically come down in recent years, it is still expensive and time-consuming to develop software for the VR systems. The cost-benefit of using VR-based training systems to train professional should be studied to quantify the financial benefits using VR in training. This is important for justifying the adaptation of VR in non-safety critical domains. It is also important to address the specific human factors issues associated with the VR technology prior to its application on educational and training settings. More research should be performed on the ergonomics, usability, quality of experience and effects of prolonged usage of VR on the users from the educational application perspective.

\subsection{Limitations of the Review}

The studies included in this review came from a wide range of training and educational contexts. Despite most studies had positive findings towards the use of VR HMDs in training, it is still hard to make a concluding statement on the effectiveness of VR HMDs in training. This is mainly due to the wide range of training applications and differing quality of research studies. Regarding the research quality, an important weakness in many studies was the measurement of learning outcomes without giving arguments for the validity of the evaluation instrument. Figure 5 summarized the type of experimental design used for studying the VR training applications. The majority of the studies utilized between-subject study design to compare VR training with other modes of training. Although case-control method was utilized in laboratory-based studies, most studies used convenience sampling. Though focused on professional training setups, majority of the studies used university students as participants. This concerns the representation of the sample to the actual end-users of the training systems. Furthermore, 
the review showed that many studies were in fact user evaluations (12 studies) of VR products that did not seek to uncover more general knowledge about learning in VR, and at the same time had an unavoidable bias towards a positive evaluation.

From the review point of view, due to omit/improper use of keywords, it is possible that relevant articles were not within the range of search of this study. Although additional articles deemed relevant were found through cross-referencing, this might be the reason for an incomplete overview of all VR training application described in the literature. Inclusion and exclusion criteria employed in the review might have some inherent limitations as well. Inclusion of grey literature such as $\mathrm{PhD}$ theses, technical reports, non-English research articles and whitepapers might have led to more exhaustive results, potentially with a larger representation from the industry.

\section{Conclusion}

In this article, we have reported the results from a systematic mapping study that reviewed the previous empirical studies focusing on the application of immersive VR for professional training. The review aimed to contribute to the knowledge of the scientific evidence on the effectiveness of VR for education and training. The findings of this review provide insights for educators and researchers into: (1) the different professional domains where VR technology was utilized for skill training (2) the types of research methods employed by previous VR studies in these domains for measuring the outcome, and (3) the types of skills trained using VR, and their related findings. The review identified a number of training situations where VR were highly valuable, such as industrial, aerospace, health care, defence, safety and emergency preparedness training. Overall, we found that majority of reviewed studies considered VR as a promising technology to enhance the learning process of trainees due to its ability to provide experiential learning, higher levels of interaction and encourage active learner participation. This combined with the availability of affordable VR headsets has led to increased interest in applying VR in educational and training settings. Still, this promise of effective training through immersive, simulation-based training should be supported by scientific evidence from testing VR training applications. As discussed in the limitations, it is still difficult to make a concluding statement on the effectiveness of VR HMDs in training. Differing quality and lack of robustness of studies point to the need for further and more rigorous research that examines the most promising uses of HMDs in authentic training contexts.

Acknowledgements The research leading to this paper has received funding from the InnoTraining research project (Project Number: 269424) funded by the Research council of Norway, and Kongsberg Digital AS.

Funding Open Access funding provided by University Of South-Eastern Norway.

\section{Compliance with Ethical Standards}

Conflict of interest The authors declare no conflict of interest.

Open Access This article is licensed under a Creative Commons Attribution 4.0 International License, which permits use, sharing, adaptation, distribution and reproduction in any medium or format, as long as you give appropriate credit to the original author(s) and the source, provide a link to the Creative Commons licence, and indicate if changes were made. The images or other third party material in this article are included in the article's Creative Commons licence, unless indicated otherwise in a credit line to the material. If material is not included in the article's Creative Commons licence and your intended use is not 
permitted by statutory regulation or exceeds the permitted use, you will need to obtain permission directly from the copyright holder. To view a copy of this licence, visit http://creativecommons.org/licenses/by/4.0/.

\section{References}

Ahlberg, G., Enochsson, L., Gallagher, A. G., Hedman, L., Hogman, C., \& McClusky, D. A., III. (2007). Proficiency-based virtual reality training significantly reduces the error rate for residents during their first 10 laparoscopic cholecystectomies. The American Journal of Surgery, 193(6), 797-804.

Anglin, J., Saldana, D., Schmiesing, A., \& Liew, S.-L. (2017). Transfer of a skilled motor learning task between virtual and conventional environments. In Paper presented at the 2017 IEEE virtual reality $(V R)$.

Aoki, H., Oman, C. M., Buckland, D. A., \& Natapoff, A. (2008). Desktop-VR system for preflight 3D navigation training. Acta Astronautica, 63(7-10), 841-847.

Babu, S. K., Krishna, S., Unnikrishnan, R., \& Bhavani, R. R. (2018). Virtual reality learning environments for vocational education: A comparison study with conventional instructional media on knowledge retention. In Paper presented at the 2018 IEEE 18th international conference on advanced learning technologies (ICALT).

Bharathan, R., Vali, S., Setchell, T., Miskry, T., Darzi, A., \& Aggarwal, R. (2013). Psychomotor skills and cognitive load training on a virtual reality laparoscopic simulator for tubal surgery is effective. European Journal of Obstetrics and Gynecology and Reproductive Biology, 169(2), 347-352.

Bliss, J. P., Tidwell, P. D., \& Guest, M. A. (1997). The effectiveness of virtual reality for administering spatial navigation training to firefighters. Presence: Teleoperators and Virtual Environments, 6(1), 73-86.

Booth, A., Sutton, A., \& Papaioannou, D. (2016). Systematic approaches to a successful literature review. Sage.

Boud, A. C., Haniff, D. J., Baber, C., \& Steiner, S. (1999). Virtual reality and augmented reality as a training tool for assembly tasks. In Paper presented at the 1999 IEEE international conference on information visualization (Cat. No. PR00210).

Bowling, S. R., Khasawneh, M. T., Kaewkuekool, S., Jiang, X., \& Gramopadhye, A. K. (2008). Evaluating the effects of virtual training in an aircraft maintenance task. The International Journal of Aviation Psychology, 18(1), 104-116.

Bowman, D. A., Koller, D., \& Hodges, L. F. (1998). A methodology for the evaluation of travel techniques for immersive virtual environments. Virtual Reality, 3(2), 120-131.

Brough, J. E., Schwartz, M., Gupta, S. K., Anand, D. K., Kavetsky, R., \& Pettersen, R. (2007). Towards the development of a virtual environment-based training system for mechanical assembly operations. Virtual Reality, 11(4), 189-206.

Burigat, S., \& Chittaro, L. (2016). Passive and active navigation of virtual environments vs. traditional printed evacuation maps: A comparative evaluation in the aviation domain. International Journal of Human-Computer Studies, 87, 92-105.

Burke, M. J., Sarpy, S. A., Smith-Crowe, K., Chan-Serafin, S., Salvador, R. O., \& Islam, G. (2006). Relative effectiveness of worker safety and health training methods. American Journal of Public Health, 96(2), 315-324.

Buttussi, F., \& Chittaro, L. (2017). Effects of different types of virtual reality display on presence and learning in a safety training scenario. IEEE Transactions on Visualization and Computer Graphics, 24(2), 1063-1076.

Caluya, N. R., Plopski, A., Ty, J. F., Sandor, C., Taketomi, T., \& Kato, H. (2018). Transferability of spatial maps: Augmented versus virtual reality training. In Paper presented at the 2018 IEEE conference on virtual reality and $3 D$ user interfaces $(V R)$.

Carlson, P., Peters, A., Gilbert, S. B., Vance, J. M., \& Luse, A. (2015). Virtual training: Learning transfer of assembly tasks. IEEE Transactions on Visualization and Computer Graphics, 21(6), 770-782.

Chen, C. J. (2010). Theoretical bases for using virtual reality in education. Themes in Science Technology Education, 2(1-2), 71-90.

Chittaro, L., \& Buttussi, F. (2015). Assessing knowledge retention of an immersive serious game vs. a traditional education method in aviation safety. IEEE Transactions on Visualization and Computer Graphics, 21(4), 529-538.

Chittaro, L., Corbett, C. L., McLean, G., \& Zangrando, N. (2018). Safety knowledge transfer through mobile virtual reality: A study of aviation life preserver donning. Safety Science, 102, 159-168. 
Chua, P. T., Crivella, R., Daly, B., Hu, N., Schaaf, R., \& Ventura, D., et al. (2003). Training for physical tasks in virtual environments: Tai Chi. In Paper presented at the IEEE Virtual Reality, 2003. Proceedings.

Clawson, D. M., Miller, M. S., Knott, B. A., \& Sebrechts, M. M. (1998). Navigational training in virtual and real buildings. In Paper presented at the proceedings of the human factors and ergonomics society annual meeting.

Clifford, R. M., Khan, H., Hoermann, S., Billinghurst, M., \& Lindeman, R. W. (2018). The effect of immersive displays on situation awareness in virtual environments for aerial firefighting air attack supervisor training. In Paper presented at the 2018 IEEE conference on virtual reality and $3 D$ user interfaces (VR).

Dalgarno, B., \& Lee, M. J. W. (2010). What are the learning affordances of 3-D virtual environments? British Journal of Educational Technology, 41(1), 10-32. https://doi.org/10.1111/j.1467-8535.2009.01038 .x.

Dang, B. K., Palicte, J. S., Valdez, A., \& O’Leary-Kelley, C. (2018). Assessing simulation, virtual reality, and television modalities in clinical training. Clinical Simulation in Nursing, 19, 30-37.

Dunn, V. (2015). Safety and survival on the fireground. New York: Fire Engineering Books.

Dwivedi, P., Cline, D., Joe, C., \& Etemadpour, R. (2018). Manual assembly training in virtual environments. In Paper presented at the 2018 IEEE 18th international conference on advanced learning technologies (ICALT).

Elbert, R., Knigge, J.-K., \& Sarnow, T. (2018). Transferability of order picking performance and training effects achieved in a virtual reality using head mounted devices. IFAC-PapersOnLine, 51(11), 686-691.

Eschenbrenner, B., Nah, F. F.-H., \& Siau, K. (2009). 3-D virtual worlds in education: applications, benefits, issues, and opportunities. In Database technologies: concepts, methodologies, tools, and applications (pp. 2595-2615): IGI Global.

Farra, S. L., Miller, E. T., \& Hodgson, E. (2015). Virtual reality disaster training: Translation to practice. Nurse Education in Practice, 15(1), 53-57. https://doi.org/10.1016/j.nepr.2013.08.017.

Fast, K., Gifford, T., \& Yancey, R. (2004). Virtual training for welding. In Paper presented at the third IEEE and ACM international symposium on mixed and augmented reality.

Fisher, S. S., McGreevy, M., Humphries, J., \& Robinett, W. (1987). Virtual environment display system. In Paper presented at the proceedings of the 1986 workshop on interactive $3 D$ graphics.

Fowler, C. (2015). Virtual reality and learning: Where is the pedagogy? British Journal of Educational Technology, 46(2), 412-422. https://doi.org/10.1111/bjet.12135.

Freina, L., \& Ott, M. (2015). A literature review on immersive virtual reality in education: state of the art and perspectives. In Paper presented at the the international scientific conference elearning and software for education.

Gallagher, A. G., Ritter, E. M., Champion, H., Higgins, G., Fried, M. P., Moses, G., et al. (2005). Virtual reality simulation for the operating room: Proficiency-based training as a paradigm shift in surgical skills training. Annals of Surgery, 241(2), 364.

Gamberini, L., Cottone, P., Spagnolli, A., Varotto, D., \& Mantovani, G. (2003). Responding to a fire emergency in a virtual environment: Different patterns of action for different situations. Ergonomics, 46(8), 842-858.

Ghanbarzadeh, R., Ghapanchi, A. H., Blumenstein, M., \& Talaei-Khoei, A. (2014). A decade of research on the use of three-dimensional virtual worlds in health care: a systematic literature review. Journal of medical Internet research, 16(2).

Grabowski, A., \& Jankowski, J. (2015a). Virtual reality-based pilot training for underground coal miners. Safety Science, 72, 310-314.

Grantcharov, T. P., Kristiansen, V. B., Bendix, J., Bardram, L., Rosenberg, J., \& Funch-Jensen, P. (2004). Randomized clinical trial of virtual reality simulation for laparoscopic skills training. British Journal of Surgery, 91(2), 146-150.

Greunke, L., \& Sadagic, A. (2016). Taking immersive VR leap in training of landing signal officers. IEEE Transactions on Visualization and Computer Graphics, 22(4), 1482-1491.

Hall, C. R., Stiles, R. J., \& Horwitz, C. D. (1998). Virtual reality for training: Evaluating knowledge retention. In Paper presented at the proceedings. IEEE 1998 virtual reality annual international symposium (Cat. No. 98CB36180).

Haller, M., Kurka, G., Volkert, J., \& Wagner, R. (1999). omVR-A safety training system for a virtual refinery. In Paper presented at the topical workshop on virtual reality and advanced human-robot systems.

Hsu, E. B., Li, Y., Bayram, J. D., Levinson, D., Yang, S., \& Monahan, C. (2013). State of virtual reality based disaster preparedness and response training. PLOS Currents Disasters. 
Huang, Y., Churches, L., \& Reilly, B. (2015a). A case study on virtual reality american football training. In Paper presented at the proceedings of the 2015 virtual reality international conference, Laval, France. https://doi.org/https://doi.org/10.1145/2806173.2806178.

Huang, Y., Churches, L., \& Reilly, B. (2015b). A case study on virtual reality American football training. In Paper presented at the proceedings of the 2015 virtual reality international conference.

Huber, T., Paschold, M., Hansen, C., Wunderling, T., Lang, H., \& Kneist, W. (2017). New dimensions in surgical training: immersive virtual reality laparoscopic simulation exhilarates surgical staff. Surgical Endoscopy, 31(11), 4472-4477. https://doi.org/10.1007/s00464-017-5500-6.

IGI consulting, I. (1992). Emerging markets for virtual reality. Boston MA: Information Gatekeepers Inc.

Jelfs, A., \& Whitelock, D. (2000). The notion of presence in virtual learning environments: What makes the environment "real." British Journal of Educational Technology, 31(2), 145-152.

Jensen, L., \& Konradsen, F. (2018). A review of the use of virtual reality head-mounted displays in education and training. Education and Information Technologies, 23(4), 1515-1529.

Jung, J., \& Ahn, Y. J. (2018). Effects of interface on procedural skill transfer in virtual training: Lifeboat launching operation study. Computer Animation and Virtual Worlds, 29(3-4), e1812.

Kinateder, M., Pauli, P., Müller, M., Krieger, J., Heimbecher, F., Rönnau, I., \& Mühlberger, A. (2013). Human behaviour in severe tunnel accidents: Effects of information and behavioural training. Transportation research Part F: Traffic Psychology and Behaviour, 17, 20-32.

Kitchenham, B. (2004). Procedures for performing systematic reviews (pp. 1-26). Keele: Keele University 33 .

Kitchenham B., \& Charters, S. (2007) Guidelines for performing Systematic Literature Reviews in Software Engineering, Version 2.3, Report EBSE-2007-01, Keele University and University of Durham.

Kitchenham, B., Pretorius, R., Budgen, D., Brereton, O. P., Turner, M., Niazi, M., \& Linkman, S. (2010). Systematic literature reviews in software engineering-A tertiary study. Information and Software Technology, 52(8), 792-805.

Kozak, J., Hancock, P., Arthur, E., \& Chrysler, S. (1993). Transfer of training from virtual reality. Ergonomics, 36(7), 777-784.

Li, C., Liang, W., Quigley, C., Zhao, Y., \& Yu, L.-F. (2017). Earthquake safety training through virtual drills. IEEE Transactions on Visualization and Computer Graphics, 23(4), 1275-1284.

Li, L., Yu, F., Shi, D., Shi, J., Tian, Z., Yang, J., \& Jiang, Q. (2017). Application of virtual reality technology in clinical medicine. American Journal of Translational Research, 9(9), 3867.

Loftin, R. B., \& Kenney, P. (1995). Training the Hubble space telescope flight team. IEEE Computer Graphics and Applications, 15(5), 31-37.

Logan, A. I. (1998). Training beyond reality. IFAC Proceedings Volumes, 31(33), 183-189.

Ludlow, B. L. (2015). Virtual reality: Emerging applications and future directions. Rural Special Education Quarterly, 34(3), 3-10.

Lugrin, J.-L., Oberdorfer, S., Latoschik, M. E., Wittmann, A., Seufert, C., \& Grafe, S. (2018). Vrassisted vs video-assisted teacher training. In Paper presented at the proceedings of the 25th IEEE virtual reality $(V R)$ conference. http://hci.uni-wuerzburg. de/download/2018-ieeevr-lugrin-vrteacher-training-poster-preprint. pdf.

Mantovani, F., Castelnuovo, G., Gaggioli, A., \& Riva, G. (2003). Virtual reality training for health-care professionals. CyberPsychology Behavior, 6(4), 389-395.

Mellet-d'Huart, D. (2009). Virtual reality for training and lifelong learning. Themes in Science and Technology Education, 2(1-2), 185-224.

Minocha, S., \& Reeves, A. J. (2010). Design of learning spaces in 3D virtual worlds: An empirical investigation of Second Life. Learning, Media and Technology, 35(2), 111-137.

Mossel, A., Froeschl, M., Schoenauer, C., Peer, A., Goellner, J., \& Kaufmann, H. (2017). VROnSite: Towards immersive training of first responder squad leaders in untethered virtual reality. In Paper presented at the 2017 IEEE virtual reality (VR).

Murcia-Lopez, M., \& Steed, A. (2018). A comparison of virtual and physical training transfer of bimanual assembly tasks. IEEE Transactions on Visualization and Computer Graphics, 24(4), 1574-1583.

Neubauer, C., Khooshabeh, P., \& Campbell, J. (2017). When less is more: Studying the role of functional fidelity in a low fidelity mixed-reality tank simulator. In Paper presented at the international conference on applied human factors and ergonomics.

Pantelidis, V. S. (2009). Reasons to Use Virtual Reality in Education and Training Courses and a Model to Determine When to Use Virtual Reality. Themes in Science and Technology Education, 2, 59-70.

Park, C.-H., Jang, G., \& Chai, Y.-H. (2006). Development of a virtual reality training system for liveline workers. International Journal of Human-Computer Interaction, 20(3), 285-303. 
Passig, D., \& Noyman, T. (2001). Training kindergarten teachers with virtual reality. In Paper presented at the IFIP world conference on computers in education.

Pausch, R., Proffitt, D., \& Williams, G. (1997). Quantifying immersion in virtual reality. In Proceedings of the 24th annual conference on Computer graphics and interactive techniques (pp. 13-18).

Pioch, N. J., Roberts, B., \& Zeltzer, D. (1997). A virtual environment for learning to pilot remotely operated vehicles. In Paper presented at the proceedings. international conference on virtual systems and multimedia VSMM'97 (Cat. No. 97TB100182).

Psotka, J. (1995). Immersive training systems-Virtual reality and education and training. Instructional Science, 23, 405-431.

Pulijala, Y., Ma, M., Pears, M., Peebles, D., \& Ayoub, A. (2018). Effectiveness of immersive virtual reality in surgical training-A randomized control trial. Journal of Oral and Maxillofacial Surgery, 76(5), 1065-1072.

Ragan, E. D., Bowman, D. A., Kopper, R., Stinson, C., Scerbo, S., \& McMahan, R. P. (2015). Effects of field of view and visual complexity on virtual reality training effectiveness for a visual scanning task. IEEE Transactions on Visualization and Computer Graphics, 21(7), 794-807.

Ragan, E. D., Sowndararajan, A., Kopper, R., \& Bowman, D. A. (2010). The effects of higher levels of immersion on procedure memorization performance and implications for educational virtual environments. Presence: Teleoperators and Virtual Environments, 19(6), 527-543.

Reid, R. D., \& Sykes, W. (1999). Virtual reality in schools: The ultimate educational technology. THE Journal, 26(7), 61-63.

Robertson, G. G., Card, S. K., \& Mackinlay, J. D. (1993). Three views of virtual reality: Nonimmersive virtual reality. Computer, $26(2), 81$.

Rose, F. D., Attree, E. A., Brooks, B. M., Parslow, D. M., \& Penn, P. (2000). Training in virtual environments: Transfer to real world tasks and equivalence to real task training. Ergonomics, 43(4), 494-511.

Sacks, R., Perlman, A., \& Barak, R. (2013). Construction safety training using immersive virtual reality. Construction Management Economics, 31(9), 1005-1017.

Sankaranarayanan, G., Wooley, L., Hogg, D., Dorozhkin, D., Olasky, J., Chauhan, S., \& Jones, D. B. (2018). Immersive virtual reality-based training improves response in a simulated operating room fire scenario. Surgical Endoscopy, 32(8), 3439-3449.

Schroeder, B. L., Bailey, S. K., Johnson, C. I., \& Gonzalez-Holland, E. (2017). Presence and usability do not directly predict procedural recall in virtual reality training. In Paper presented at the international conference on human-computer interaction.

Shen, J., \& Eder, L. B. (2009). Intentions to use virtual worlds for education. Journal of Information Systems Education, 20(2), 225.

Singer, M. J., Allen, R. C., McDonald, D. P., \& Gildea, J. P. (1997). Terrain appreciation in virtual environments: Spatial knowledge acquisition. Retrieved from

Stansfield, S., Shawver, D., Sobel, A., Prasad, M., \& Tapia, L. (2000). Design and implementation of a virtual reality system and its application to training medical first responders. Presence: Teleoperators and Virtual Environments, 9(6), 524-556.

Stedmon, A. W., \& Stone, R. J. (2001). Re-viewing reality: Human factors of synthetic training environments. International Journal of Human-Computer Studies, 55(4), 675-698.

Stevens, J. A., \& Kincaid, J. P. (2015). The relationship between presence and performance in virtual simulation training. Open Journal of Modelling and Simulation, 3(2), 41-48. https://doi.org/10.4236/ojmsi .2015 .32005 .

Sutherland, I. E. (1965). The ultimate display. Information Processing 1965: Proceedings of the IFIP Congress, pp. 506-508.

Tanaka, E. H., Paludo, J. A., Bacchetti, R., Gadbem, E. V., Domingues, L. R., \& Cordeiro, C. S., et al. (2017). Immersive virtual training for substation electricians. In Paper presented at the 2017 IEEE virtual reality $(V R)$.

Tate, D. L., Sibert, L., \& King, T. (1997). Using virtual environments to train firefighters. IEEE Computer Graphics and Applications, 17(6), 23-29.

Vaughan, N., Gabrys, B., \& Dubey, V. N. (2016). An overview of self-adaptive technologies within virtual reality training. Computer Science Review, 22, 65-87.

Vora, J., Nair, S., Gramopadhye, A. K., Duchowski, A. T., Melloy, B. J., \& Kanki, B. (2002). Using virtual reality technology for aircraft visual inspection training: Presence and comparison studies. Applied Ergonomics, 33(6), 559-570.

Waller, D., Hunt, E., \& Knapp, D. (1998). The transfer of spatial knowledge in virtual environment training. Presence, 7(2), 129-143.

Witmer, B. G., Bailey, J. H., Knerr, B. W., \& Parsons, K. C. (1996). Virtual spaces and real world places: Transfer of route knowledge. International Journal of Human-Computer Studies, 45(4), 413-428. 
Witmer, B. G., \& Singer, M. J. J. P. (1998). Measuring presence in virtual environments: A presence questionnaire. Presence, 7(3), 225-240.

Xu, J., Tang, Z., Yuan, X., Nie, Y., Ma, Z., Wei, X., \& Zhang, J. (2018). A VR-based the emergency rescue training system of railway accident. Entertainment Computing, 27, 23-31.

Yang, U., \& Kim, G. J. (2002). Implementation and evaluation of "just follow me": An immersive, VRbased, motion-training system. Presence: Teleoperators and Virtual Environments, 11(3), 304-323.

Yoganathan, S., Finch, D., Parkin, E., \& Pollard, J. (2018). 360 virtual reality video for the acquisition of knot tying skills: A randomised controlled trial. International Journal of Surgery, 54, 24-27.

Zhang, H. (2017). Head-mounted display-based intuitive virtual reality training system for the mining industry. International Journal of Mining Science and Technology, 27(4), 717-722.

Zhang, K., Suo, J., Chen, J., Liu, X., \& Gao, L. (2017). Design and implementation of fire safety education system on campus based on virtual reality technology. In Paper presented at the 2017 federated conference on computer science and information systems (FedCSIS).

Publisher's Note Springer Nature remains neutral with regard to jurisdictional claims in published maps and institutional affiliations. 